\title{
Coupling Between Spins and Phonons Towards Ferroelectricity in Magnetoelectric Systems
}

\author{
J. Agostinho Moreira and A. Almeida \\ IFIMUP-IN, Institute of Nanosciences and Nanotechnologies \\ Departamento de Física e Astronomia da Faculdade de Ciências da Universidade do Porto \\ Portugal
}

\section{Introduction}

Materials, which are either electrically or magnetically polarizable, have attracted the interest of the scientific community for long, due to both their potential technological applications and the emergent fundamental questions associated with the mechanisms tailoring their pertinent physical properties. ${ }^{1}$

Among them, a class of materials exists, exhibiting at least two ferroic ground states, which are known as multiferroic materials. ${ }^{2}$ Another class is characterized by the emergence of a magneto-electric coupling, where multiferroicity is not even necessary to be present. This sort of materials yields the possibility to control the magnetic (polar) state through an applied electric (magnetic field). One of the most relevant examples is its use to processing of barriers and interfaces to spintronic devices, enabling to implement electrically controlled spin transport, but other interesting applications are currently under investigation. ${ }^{3-11}$

Unfortunately though, either magnetoelectric coupling coefficient is rather small, or the range of temperatures it occurs is far way from room temperature, precisely where devices are requested to have their working range. Based on theoretical and experimental results, an intensive research to designing new magnetoelectric materials has been undertaken aimed at overcoming the aforesaid drawbacks. ${ }^{12}$ On designing new systems, their crystal structure has been addressed concerning both lattice and geometrical arrangements of their atomic or molecular constituents. This is particularly the case of the orthorhombic rare-earth manganites belonging to the $\mathrm{GdFeO}_{3}$ type structure. ${ }^{13}$ In this sort of structure, rare-earth site alteration drives the magnitude of the orthorhombic distortion, so-called cooperative $\mathrm{GdFeO}_{3}$-type distortion, wherein the increase of the Mn-O1-Mn bond angle of the $\mathrm{MnO}_{6}$ octahedra yields an increasing of the orthorhombic distortion, whenever a smaller-sized rare-earth ion is used.13 Thus, by handling the Mn-O1-Mn bonds angle between consecutive $\mathrm{MnO}_{6}$ octahedra, drastic changes of their phase diagrams can be reached.13 As it will be presented further below, this is highly predictable, since changing the Mn-O1-Mn bond angle directly modifies the balance between the competitive ferromagnetic and antiferromagnetic exchange interactions, existing in these materials. This is the background mechanism of magnetically induced ferroelectricity, in some rare-earth manganites.

Contrarily to well know ferroelectrics, like $\mathrm{BaTiO}_{3}$, where hybridization between the titanium $3 \mathrm{~d}$ states and the oxygen $2 \mathrm{p}$ states is at the very origin of ferroelectricity, in 
magnetoelectric rare-earth materials it seems to stem from the emergence of spin-lattice interactions in modulated off-centre symmetric magnetic structures, which is in favour for the improper nature of the ferroelectric ground state. For some magnetoelectric materials, it has been proposed that ferroelectricity is originated from a variety of spiral magnetic structures, and can be explained in terms of the inverse Dzyaloshinski-Morya model. ${ }^{14-16}$ On the grounds of this model, the interaction between spins, arranged in a modulated structure, deform the crystal lattice, yielding the loss of the spatial inversion centre and, thus, enabling the emergence of electric dipoles. As the electric polarization arises from lattice distortions, the study of the coupling between spins and phonons is of most relevant matter in systems, wherein magnetic and ferroelectric properties are coupled. Consequently, from both fundamental and application point of views, a deeper understanding of spin-phonon coupling remains a very important issue.

The most studied magnetoelectric materials are the orthorhombic rare-earth manganites, such as $\mathrm{EuMnO}_{3}, \mathrm{GdMnO}_{3}, \mathrm{TbMnO}_{3}$ and $\mathrm{DyMnO}_{3} \cdot{ }^{17-23}$ These systems exhibit rather interesting phase diagrams, with magnetically driven ferroelectric phase transitions, for compounds with the lighter rare-earth ions, or magnetically switching of electric polarization, for those ones with the heavier rare-earth ions. These materials behave differently since, by changing rare-earth ion size, the Mn-O1-Mn bond angle changes accordingly and hence the balance between ferromagnetic and antiferromagnetic interactions. Unfortunately, the change of rare-earth ions also brings changes to the magnetic state through its own magnetic characteristics. If it is required to comprehend just the effect of ion radius size, the solid solution obtained by A-site substitution in europium manganite with a non-magnetic ion, with a radius smaller than europium will ensure the aforesaid requisites. Since neither yttrium nor europium ions possess magnetic moment, interchanging them does not change the total magnetic moment. It stems only from the $\mathrm{Mn}^{3+}$ ions. From the reasoning presented to above, it is apparent why Y-doped $\mathrm{EuMnO}_{3}$ system has been extensively studied to date. ${ }^{24-32}$

Several studies of the lattice dynamics by using Raman and infrared spectroscopies in Ydoped $\mathrm{EuMnO}_{3}$, have revealed a coupling between magnetic excitations and phonons, through anomalous temperature behaviour of the phonon parameters or absorption across the magnetic phase transitions. ${ }^{33-38}$ However, an overview of the experimental results in order to evidence the main mechanisms underlying the spin-phonon coupling is still missing.

In this book chapter, we will present a detailed review of the main theoretical and experimental results concerning the lattice dynamics and spin-phonon coupling in the case study: $\mathrm{Eu}_{1-x} \mathrm{Y}_{x} \mathrm{MnO}_{3}$ for $0 \leq x \leq 0.5$, where the structure is orthorhombic with the Pbnm space group. The main goal is to understand the mechanisms of coupling between magnetic excitations and phonons yielding the magnetically switching of ferroelectricity in spiral magnets and, in this way, the very origin of the ferroelectric ground state in these compounds.

\section{Theoretical background}

Important advances have been achieved towards a fundamental understanding of the stabilization of ferroelectric ground states in magnetoelectrics through experimental observation and investigation of the coupling mechanism between the ferroelectric and magnetic orders. Some examples involve the use of second harmonic light generation, 
dielectric and magnetic measurements or structural investigation. In contrast, very little is known about the behaviour of phonons in magnetoelectrics, although studies of phonons have in the past played a crucial role in the understanding of classic ferroelectrics. Phonons are also known to be altered by spin correlations thus offering a complementary and maybe even unique powerful tool to investigate coupling mechanism in magnetoelectrics. ${ }^{39-41}$

Before going into details of phonon analysis and their coupling with spins, let us summarize some aspects associated with the origin and main characteristics of spin structure in orthorhombic rare-earth manganites. The main crystalline motif of their structure is the existence of $\mathrm{MnO}_{6}$ octahedra, which run over chains by sharing apical oxygen ions. The $3 \mathrm{~d}$ electronic orbitals of the transition $\mathrm{Mn}^{3+}$ ions yield a rather multifaceted correlation between spin arrangements and crystalline structure, which is at the very origin of the broad range of physical properties observed in rare-earth manganites. In an ideal cubic crystal field environment, the $3 d \mathrm{Mn}^{3+}$ orbitals are splitted into two degenerate higher energy states, called $d\left(\mathrm{x}^{2}-\mathrm{y}^{2}\right)$ and $d\left(3 \mathrm{z}^{2}-\mathrm{z}^{2}\right)$, with e symmetry, and three degenerate lower energy states, $\left(d_{\mathrm{xy}}, d_{\mathrm{xz}}, d_{\mathrm{z}}\right)$, with $\mathrm{t}_{2 \mathrm{~g}}$ symmetry. Since e $\mathrm{g}$ orbitals are much closer to the $2 p$ oxygen electronic orbitals, Coulomb interaction yields a partial degeneracy lifting of the $3 d$ orbitals. Moreover, if the magnitude of the crystal field is much larger than spin-orbit coupling, a quenching of the orbital angular momentum occurs, reflecting the actual spin structure in rare-earth manganites. 42,43

Over Coulomb interaction, the $3 d$ electrons of $\mathrm{Mn}^{3+}$ ions can assure different kinds of interactions. Whilst Hund and direct interactions favour ferromagnetic ordering of spins, Hubbard tends to align spins antiferromagnetically. Moreover, orbital degeneracy is at the origin of some types of superexchange interactions. From the substantial overlapping of these latter orbitals with $2 p$ oxygen ones, a strong superexchange interaction is obtained. Contrarily, due to the orthogonal spatial orientation of the $t_{2 g}$ orbitals against the $2 p_{\sigma}$ oxygen orbitals and just a reduced overlapping with the $2 p_{\pi}$ ones, weak superexchange interactions are thus expected. ${ }^{42,43}$

In orthorhombic rare-earth manganites a significant distortion occurs associated with $\mathrm{MnO}_{6}$ octahedra, which is known as the Jahn-Teller effect.44,45 This distortion, which occurs along the molecular fourfold axes, has the effect of lifting both orbital and electronic degeneracies, leading to a decrease of the total energy. ${ }^{42,43}$

The coupling between spins and lattice has become one of the major mechanisms to understand the interplay between magnetism and ferroelectricity occurring in rare-earth manganites with intermediate-range magnitudes of the $\mathrm{GdFeO}_{3}$ distortion, as it is the case of $\mathrm{ReMnO}_{3}, \mathrm{Re}=\mathrm{Eu}, \mathrm{Gd}, \mathrm{Tb}$, and Dy. The so-called magnetoelectric effect has been understood in the scope of both phenomenological and microscopic theories. In the former case, contributions first from Landau and latter from Dzyaloshinskii and Moriya (DM) were presented, wherein symmetry conditions imposed by the magnetic point group are essential issues for understanding the emergence of the magnetoelectric effect. ${ }^{46-48}$ In particular, both time and inversion symmetries are key issues for controlling the ME effect. Microscopic quantum theory of the ME effect has been developed by I. A. Sergienko et al ${ }^{16}$ and Katsura et al. 49 These latter authors showed that ME effect and spin current are related to one another in non-linear spin structures, like spiral-like states. In fact, as both electric polarization and spin current belong to the same symmetry class, it is expected that are coupled together. The coupling was demonstrated by considering a cluster scheme with two transition metal ions intermediated by an oxygen atom shown in Figure 1. 


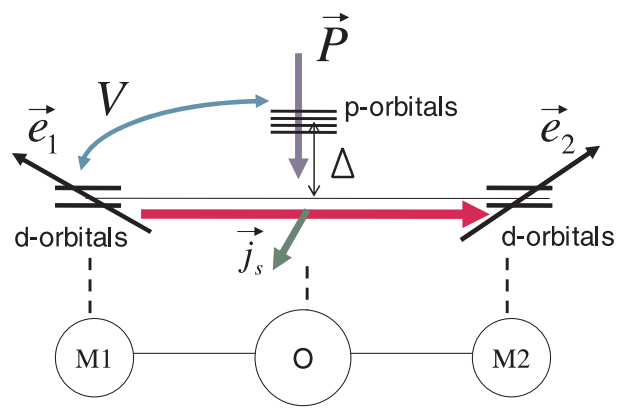

Fig. 1. The cluster model with two transition metal ions M1 and M2, and an oxygen atom between them. Reprinted figure from Ref. 49. Copyright (2005) by the American Physical Society.

By assuming the overlapping between the electronic wave functions of two adjacent sites $i$ and $i+1$ with mutually canted spins, $\boldsymbol{S}_{i}$ and $\boldsymbol{S}_{i+1}$, the inverse Dzyaloshinskii-Moriya model yields a local polarization $p_{i}$, given by: 50

$$
\boldsymbol{p}_{i}=A \boldsymbol{e}_{i, i+1} \times\left(\boldsymbol{S}_{i} \times \boldsymbol{S}_{i+1}\right) .
$$

$\boldsymbol{e}_{i, i+1}$ stands for the unit vector linking both sites, being $A$ a constant reflecting spin-orbit interactions and spin-exchange. Thus, the cycloidal spin arrangement in rare-earth manganites enables an electric polarization to emerge, obtained by summing all of the local polarization $\boldsymbol{p}_{i}$. It comes out from equation (1) that the polarization lays in the cycloidal plane. As a consequence, in rare-earth manganites, the ferroelectric polarizations expected along $a$ - and $c$-direction lay in the $a b$ - and $c b$-cycloidal planes, respectively (Figure 2).

(a)

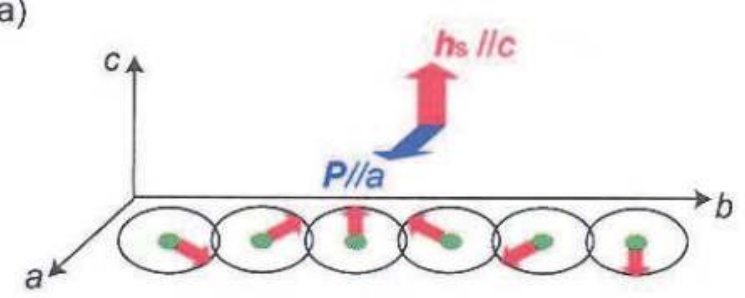

(b)

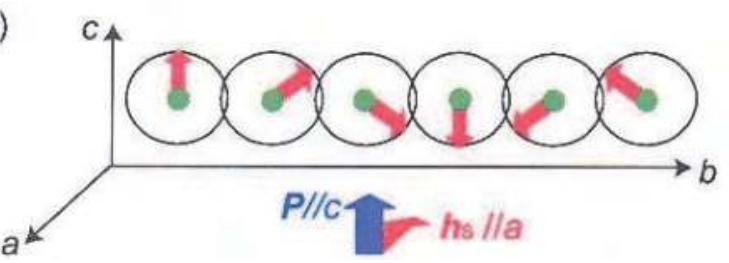

Fig. 2. Spatial scheme of the DM polarization against $\boldsymbol{h}_{i}=\sum_{j} \boldsymbol{S}_{\boldsymbol{j}} \times \boldsymbol{S}_{\boldsymbol{j}+\mathbf{1}}$ for (a) $a b$-cycloidal spin plane and (b) bc-cycloidal spin plane. Reprinted figure from Ref. 50. Copyright (2009) by the American Physical Society. 
The inverse DM model has been successfully tested both theoretically and experimentally in a variety of magnetoelectric rare-earth manganite systems. ${ }^{15,16,51,52}$ The emergence of inverse$\mathrm{DM}$ induced polarization in rare-earth manganites depends on the magnitude of the $\mathrm{GdFeO}_{3}$ distortion, characterized by the amplitude of the Mn-O1-Mn bond angle or the rareearth ionic radius size. The following examples show that increasing (decreasing) the $\mathrm{GdFeO}_{3}$ distortion (Mn-O1-Mn bond angle or the rare-earth radius size) enhances the inverse-DM magnetically induced polarization. The first example is the emergence of an electric polarization along the $c$-direction when changing from $\mathrm{GdMnO}_{3}$ to $\mathrm{TbMnO}_{3}$ and $\mathrm{DyMnO}_{3}$, which is associated with the increase of the $\mathrm{GdFeO}_{3}$ distortion due to ionic radius size decrease. ${ }^{53,54}$ Further examples can be found in $\mathrm{Gd}_{1-x} \mathrm{~Tb}_{x} \mathrm{MnO}_{3}{ }^{55,56}$ and $\mathrm{Eu}_{1-x} \mathrm{Y}_{x} \mathrm{MnO}_{3}$ 24,26,31,57,58 solid solutions.

Changes of the polarization state are obtained by continuously control the $\mathrm{GdFeO}_{3}$ distortion by increasing dopant content, decreasing in this way the ionic radius size. In fact, above a certain doping level a long-range ferroelectric ground state is stabilized at low temperatures associated with an $a b$-cycloidal spin state, where electric polarization emerges along the $a$-crystallographic direction. For larger dopant concentration the cycloidal $c b$-plane spontaneously flops at higher temperature to the $a b$-plane at lower temperatures, along with a rotation of the electric polarization from the $c$ - to the $a$-direction. ${ }^{26}$

The examples referred to above undoubtedly evidence the role of the $\mathrm{GdFeO}_{3}$ distortion to inducing electric polarization via inverse DM interaction. Moreover, it is has been established that this distortion has a crucial effect on the balance between the ferromagnetic superexchange interaction, associated with the next-nearest (NN) spins, and the antiferromagnetic superexchange one of the next-next nearest (NNN) spins. ${ }^{13,50}$ Increasing the $\mathrm{GdFeO}_{3}$ distortion enhances the antiferromagnetic against the ferroelectric interactions. This mechanism apparently yields the reduction of the AFM(A) phase transition temperature with decreasing Mn-O1-Mn bond angle in orthorhombic rare-earth manganites, and the emergence of the AFM ordering or spiral spin ordering, where ferroelectric ground states can be stabilized.

Several attempts have been made in order to develop microscopic spin models to understand the phase diagrams of rare-earth manganites. ${ }^{13,16,59-62}$ The essence of magnetoelectric properties can be described if they are mapped onto a frustrated classical $\mathrm{S}=2$ Heisenberg model on a cubic lattice, with FM NN $\left(J_{a b}\right)$, and AFM NNN $\left(J_{2}\right)$ exchange interactions acting in the $a b$-plane, and AFM NN $\left(J_{c}\right)$ exchange interaction along the $c$-direction, as it is shown in figure 3. In the Hamiltonian the $\mathrm{Mn} S=2$ spins are considered as classical vectors expressed as $\boldsymbol{S}_{i}=\left(\sqrt{S^{2}-S_{c}^{2}} \cos \theta_{i}, \sqrt{S^{2}-S_{c}^{2}} \sin \theta_{i}, S_{c}\right)$, with their components along $a, b$, and $c$ axes of the Pbnm space group.

The Hamiltonian includes four terms involving superexchange and DM interactions, and contributions from both single ion and cubic anisotropies, expressed as follows: 50

$$
H=H_{e x}+H_{D M}+H_{s i a}+H_{c u b},
$$

wherein:

$$
\begin{gathered}
H_{e x}=-J_{a b} \sum_{<i, j>}^{x, y} \boldsymbol{S}_{i} \cdot \boldsymbol{S}_{j}+J_{2} \sum_{<i, j>}^{b} \boldsymbol{S}_{i} \cdot \boldsymbol{S}_{j}+J_{c} \sum_{<i, j>}^{c} \boldsymbol{S}_{i} \cdot \boldsymbol{S}_{j}, \\
H_{D M}=\sum_{<i, j>} \boldsymbol{d}_{i j}^{\alpha} \cdot\left(\boldsymbol{S}_{i} \times \boldsymbol{S}_{j}\right), \\
H_{\text {sia }}=D \sum_{i} S_{\zeta i}^{2}+E \sum_{i}(-1)^{i_{x}+i_{y}}\left(S_{\zeta i}^{2}-S_{\eta i}^{2}\right),
\end{gathered}
$$




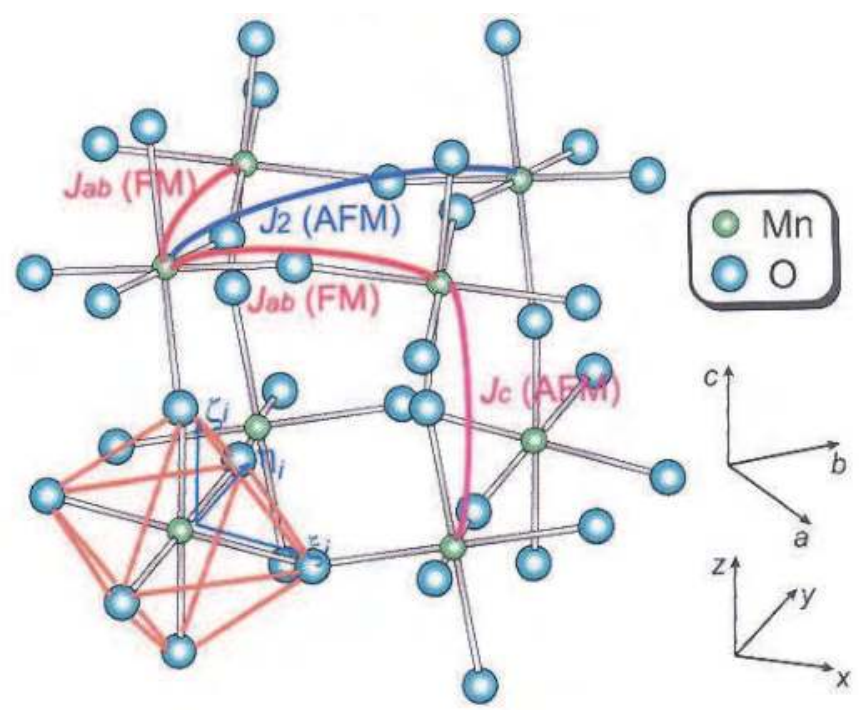

(a)

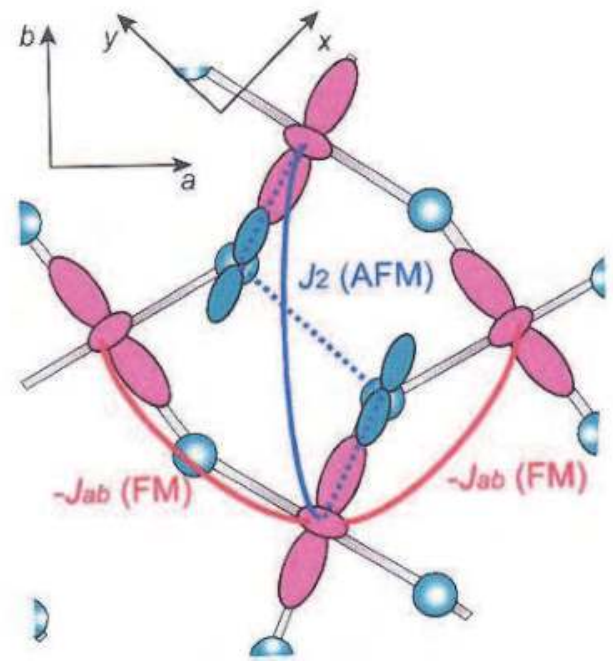

(b)

Fig. 3. (a) Exchange interactions in rare-earth manganites. $J_{a b}$-FM interactions on the Mn-Mn bonds along the cubic $x$ - and $y$-axes, $J_{2}$-AFM interactions on the in-plane diagonal Mn-Mn bonds along the orthorhombic $b$-axis, and $J_{c}$-AFM interactions on the Mn-Mn bonds along the $c$-axis. (b) $e_{g}$ occupied orbitals configuration and both $J_{a b}$ and $J_{2}$ interactions (solid lines) in the $a b$-plane. A dashed line marks $J_{2}$ interaction path, via two oxygen $2 p$ orbitals. Reprinted figure from Ref. 50. Copyright (2009) by the American Physical Society. 
and

$$
H_{c u b}=\frac{a}{S(S+1)} \sum_{i}\left(S_{x i}^{4}+S_{y i}^{4}+S_{z i}^{4}\right) .
$$

$i_{x}, i_{y}$ and $i_{z}$ are the coordinates of the $i$-th manganese ion along the $x, y$, and $z$ cubic axes.

The superexchange term consists of NN FM $J_{a b}$, NNN AFM $J_{2}$, and AFM NN $J_{c}$ interactions. $J_{a b}$ is associated with the AFM arrangement of $e_{g}$ orbitals in the $a b$-plane, while $J_{2}$ stems from AFM exchange between the two Mn $e_{g}$ orbitals in the $b$-direction, along the path defined by the two $2 p$ oxygen orbitals. AFM $J_{c}$ comes from ferro-orbital stacking along the $c$-direction. As in manganites $J_{a b}$ and $J_{c}$ are comparable in magnitude, and $J_{2}$ is strongly dependent on $\mathrm{GdFeO}_{3}$ distortion, it is evident the important role played by changing the Mn-O1-Mn bond to alter the magnetic properties of rare-earth manganites. Thus, it is expected that by increasing $J_{2}$ against $J_{a b}$, the $\mathrm{GdFeO}_{3}$ distortion will tend to stabilize spiral spin ordering with ferroelectric properties. This is in perfect agreement with the experimental results obtained in the rare-earth manganite systems referred to above.

The second term of the Hamiltonian (Eq. 4) concerns the DM interactions. ${ }^{47,48,63}$ The $\boldsymbol{d}_{i j}^{\alpha}$ vector regards the $\mathrm{Mn}(\mathrm{i})-\mathrm{O}-\mathrm{Mn}(\mathrm{j})$ bond along the a cubic axis $(\alpha=x, y, z)$. Figure 4 shows the DM in-plane and out-of-plane vectors in the vicinity of the $\mathrm{Mn}(\mathrm{i})-\mathrm{O}-\mathrm{Mn}(\mathrm{j})$.

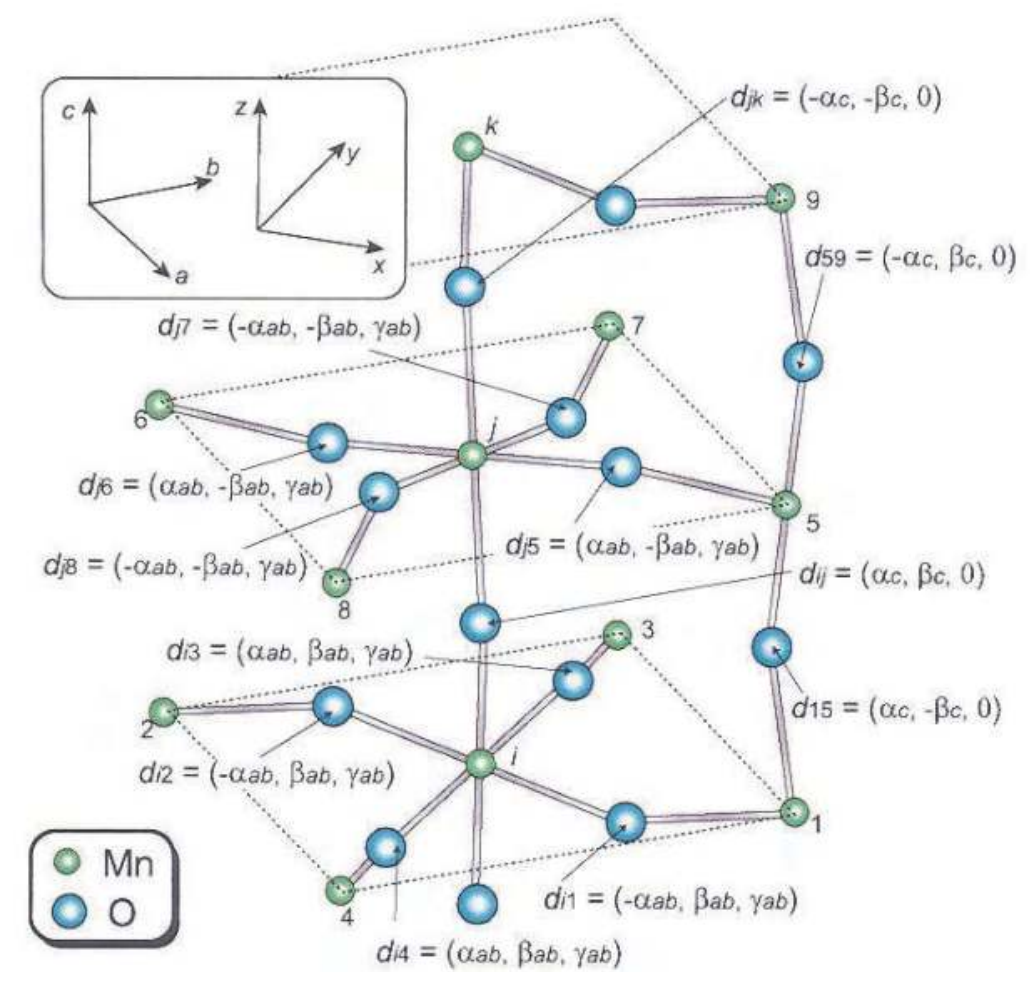

Fig. 4. DM vectors around the $\mathrm{Mn}(\mathrm{i})-\mathrm{O}-\mathrm{Mn}(\mathrm{j})$ bond, expressed as a function of the parameters $\alpha_{a b}, \beta_{a b} \gamma_{a b}, \alpha_{c}$, and $\beta_{c}$. Reprinted figure from Ref. 50. Copyright (2009) by the American Physical Society. 
From the spatial orientation of the $e_{g}$ orbitals it can be reckoned that while the DM vectors regarding in-plane $\mathrm{Mn}-\mathrm{O}-\mathrm{Mn}$ bonds are almost perpendicular to the plane, those corresponding to out-of-plane bonds are not. Recent calculations yield a magnitude three times larger for the out-of-plane DM vector relative to the in-plane one. It is worthwhile to note that $a_{c}$ has the same sign in each plane, though alternating along de $c$ axis. Due to spin canting in the AFM(A) phase, a week ferromagnetism is thus expected along $c$ axis, in good agreement with earlier results. ${ }^{26,64}$

The single-ion anisotropy contribution is determined by the octahedron environment of the manganese ion. The first term of equation 5 implies that the $c$-axis becomes a hard magnetization axis, as $s_{i}$ is directed mainly along $c$. Contrarily, the second term evidences the existence of local magnetization axes along $\xi_{i}$ and $\eta_{i}$, alternatively located in the $a b$ plane. The cubic anisotropy term reflects the cubic anisotropy, which stems from the nearly cubic symmetry of the perovskite lattice. The contribution from orthorhombic distortion is not taken into account, since its magnitude is comparatively very small.

Earlier experimental results, which were aimed at studying the magnetic, electric and magnetoelectric properties of rare-earth manganites, reveal that the $\mathrm{GdFeO}_{3}$ distortion plays a major role by enhancing the AFM exchange interactions $J_{2}$ against the FM ones $J_{a b}$. Thus, it is challenging to trace the $\left(\mathrm{T}, J_{2}\right)$ phase diagrams in order to understand those obtained experimentally as a function of either the rare-earth radius size or the concentration of dopant ion. Figure 5 shows the $\left(T, J_{2}\right)$ phase diagram obtained on the grounds of this model for $a_{c}=0.30 \mathrm{meV}$, and tends to reproduce the main characteristics revealed by a variety of experimental $(\mathrm{T}, x)$ phase diagrams earlier presented for $\mathrm{Eu}_{1-x} \mathrm{Y}_{x} \mathrm{MnO}_{3 .}{ }^{24,26,31}$

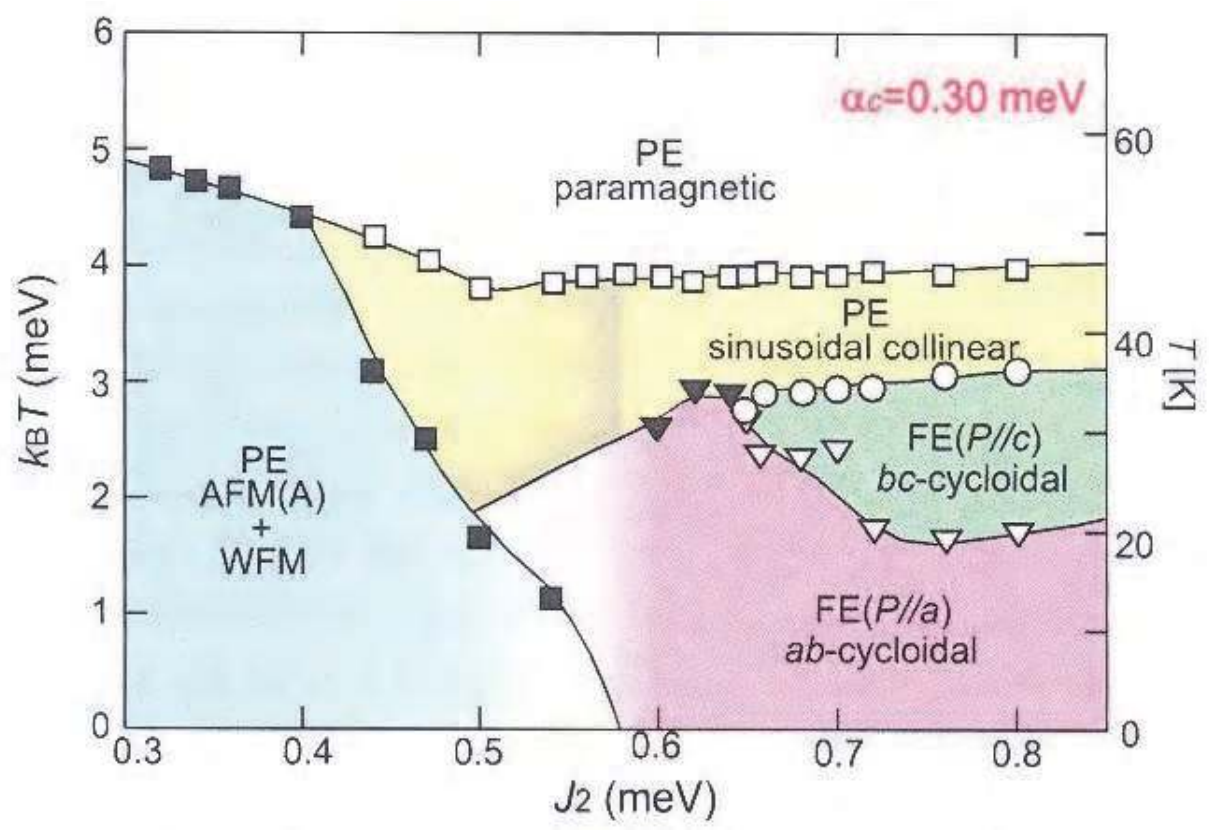

Fig. 5. Theoretical $\left(\mathrm{T}, J_{2}\right)$ phase diagram obtained for $a_{c}=0.30 \mathrm{meV}$. Reprinted figure from Ref. 50. Copyright (2009) by the American Physical Society. 
Except for some distinctive details regarding the trace of the phase boundaries, the diagram shown in Figure 5 reproduces quite well the phase arrangement obtained experimentally. WFM+AFM(A) phase emerges for lower values of $J_{2}$. For increasing $J_{2}$ values, AFM modulated phases are stabilized, where modulation is a consequence of DM interaction, here determined by $a_{c}=0.30 \mathrm{meV}$. The modulation of the AFM phases is needed for stabilizing the ferroelectric ground state.

It is also worth to note the flop of the polarization from $\mathrm{P}_{c}$ at high temperatures to $\mathrm{P}_{a}$ at low temperatures for higher values of $J_{2}$. This flop is though puzzling. Since the $c$ axis in rareearth manganites is always the magnetization hard axis due to the $H_{s i a}=D \sum_{i} S_{\zeta i}^{2}$ single-ion anisotropy, it is expected a higher energy for the $b c$-cycloidal spin state than for the $a b$ one. The reason for the stabilization of the $b c$-cycloidal spin state for high $J_{2}$ values stems from considering the energy balance $\left|\Delta E_{D M}^{b c}-\Delta E_{D M}^{a b}\right|$ due to DM term for both $b c$ - and $a b$-spin states. In fact, this balance dominates for high $J_{2}$ values the energetic disadvantage stemming from the hard $c$-magnetization axis. This means that the $a b$ - and $b c$-cycloidal spins states are actually stabilized by single-ion anisotropy or DM interactions.

Moreover, as the spins in the bc-cycloidal state are mainly associated with $a$ component of $\mathrm{DM}$ vectors on the out-of-plane Mn-O-Mn bonds, it is expected that $a_{c}$ becomes an important parameter to determine the relative temperature range of both $b c$ - and $a b$-spin states. It can be shown by increasing $a_{c}$ up to $0.38 \mathrm{meV}$ that the $\mathrm{Gd}_{1-x} \mathrm{~Tb}_{x} \mathrm{MnO}_{3}$ phase diagram can be reproduced, where for high values of $J_{2}$ just the $b c$-cycloidal spin state is stabilized in good agreement with earlier experimental results. Contrarily to earlier results, it is also evidenced that the flop of the cycloidal spin plane is quite independent of the $f$-electron moments, as it becomes clear from the $\left(\mathrm{T}, J_{2}\right)$ phase diagrams of $\mathrm{Gd}_{1-\mathrm{x}} \mathrm{Tb}_{\mathrm{x}} \mathrm{MnO} 3$ and $\mathrm{Eu}_{1-x} \mathrm{Y}_{x} \mathrm{MnO} 3$ systems. ${ }^{24,26,31}$

Additionally, by analysing the single-ion anisotropies, it can be reckoned that the $\mathrm{GdFeO}_{3}$ distortion energetically favours the orientation of the spins along the $b$ axis in both the AFM(A) and sinusoidal collinear phases.

\section{Case study: $\mathrm{Eu}_{1-\mathrm{x}} \mathrm{Y}_{x} \mathrm{MnO3}(0<x<0.55)$}

The coupling between spin and phonons has been observed in a broad range of materials, exhibiting ferromagnetic, antiferromagnetic, magnetoresistive, or superconducting properties. Many of them do not present magnetoelectric effect, evidencing that the existence of that coupling does not necessary lead to the emergence of this effect. ${ }^{65-68}$ Thus, if we aim at assessing the role of spin-phonon coupling to stabilize ferroelectric ground states, it will be undertaken in materials that exhibit magnetoelectric coupling. Orthorhombic rareearth manganites are actually good candidates as magnetoelectricity can be gradually induced by simply changing the rare-earth ion.13,16,17,50 This is the case of rare-earth ions passing from $\mathrm{Nd}, \mathrm{Sm}, \mathrm{Eu}, \mathrm{Gd}, \mathrm{Tb}$, to $\mathrm{Dy}$, which are quite suitable to study the way the magnetoelectric effect correlates with spin-phonon coupling. However, along with the change of the ionic radius size, an unavoidable change of the total magnetic moment will occur, due to the different magnitude of the magnetic moment of each rare-earth ion. The best way to have just the change of one variable is to preserve the magnetic moment by choosing a system that does not involve any other magnetic moments than those that stem from the manganese ions. There is at least one system that fulfils these requirements. The solid solution obtained by introducing yttrium ions at the A-site of $\mathrm{EuMnO}_{3}$. Since both europium and yttrium ions do not possess any magnetic moment by interchanging them the 
total magnetic moment remains constant. Most interesting is then what really changes? As yttrium has a smaller radius than europium by increasing yttrium content the effective Asite radius decreases accordingly. We have then a system worth to be studied, where by decreasing just the A-site effective site and consequently decreasing the Mn-O1-Mn bond angle, it will directly act on the balance of the ferromagnetic and antiferromagnetic interactions, tailoring in the way the phase diagram of the system. One of the consequences is the stabilization of spiral incommensurate antiferromagnetic spin structures, enabling the emergence of ferroelectric ground states on the basis of the DM model.

From the phase diagram presented to above it is reckoned that the different compositions for $x$ less than 0.5 can be gathered in several sets with specific physical properties. Thus, the experimental data obtained will be shown by taking this division into sets in account. But before going into it, let us summarize the state-of-art of the yttrium doped $\mathrm{EuMnO}_{3}$ system.

\subsection{General considerations about the phase diagram of $\mathrm{Eu}_{1-x} \mathrm{Y}_{x} \mathrm{MnO}_{3}, x<0.55$}

The possibility of systematic and fine tuning of the A-site size, without increasing the magnetic complexity arising from the rare-earth ion, is achieved by the isovalent substitution of the trivalent $\mathrm{Eu}^{3+}$ ion by $\mathrm{Y}^{3+}, \mathrm{Eu}_{1-x} \mathrm{Y}_{x} \mathrm{MnO}_{3}$, with $x<0.55$. This allows us for a continuous variation of the Mn-O1-Mn bond angle, which is associated with the development of the complex magnetic ground states and ferroelectric phases.

The main features of the phase diagram of $\mathrm{Eu}_{1-x} \mathrm{Y}_{x} \mathrm{MnO}_{3}$, with $0 \leq x<0.55$, has been described on the grounds of competitive NN ferromagnetic and NNN antiferromagnetic interactions, along with single-ion anisotropy and the Dzyaloshinsky-Morya interaction. ${ }^{50}$ Therefore, this system exhibits a rich variety of phase transitions from incommensurate to commensurate antiferromagnetic phases, some of them with a ferroelectric character, depending on the magnitude of $x$ substitution.

We should highlight the importance of this result as it definitely confirms assumptions forwarded in previously published works carried out in orthorhombically distorted rareearth maganites.17-26 What makes them a very interesting set of materials is the fact that they share a common $\mathrm{GdFeO}_{3}$-distortion, where the tilt angle of the $\mathrm{MnO}_{6}$ octahedra becomes larger when the rare-earth radius decreases. This behaviour is illustrated in Figure 6 for several undoped rare-earth manganites and the $\mathrm{Eu}_{1-x} \mathrm{Y}_{x} \mathrm{MnO}_{3}$ doped system. ${ }^{31}$ As it can be seen for undoped rare-earth manganites, by decreasing the ionic radius size, the Mn-O1$\mathrm{Mn}$ bond angle decreases almost linearly. However, for the $\mathrm{Eu}_{1-x} \mathrm{Y}_{x} \mathrm{MnO}_{3}$ system, a significant deviation from the linear behaviour observed for undoped manganites, is detected. It is worthwhile to note that a much steeper slope is observed for the $\mathrm{Eu}_{1-x} \mathrm{Y}_{x} \mathrm{MnO}_{3}$ system. Since the slope of the Mn-O1-Mn bond angle as a function of $x$ scales with the degree of competition between both the NN neighbour ferromagnetic and the NNN antiferromagnetic exchanges in the basal $a b$-plane, its phase diagram has then to exhibit very unique features, which distinguish the $\mathrm{Eu}_{1-x} \mathrm{Y}_{x} \mathrm{MnO}_{3}$ system from the others. Such features are apparent out from earlier phase-diagrams. ${ }^{24,26}$

Ivanov et al58, Hemberger et al24, and Yamasaki et al26 have proposed $(x, \mathrm{~T})$ phase diagrams, for $\mathrm{Eu}_{1-x} \mathrm{Y}_{x} \mathrm{MnO}_{3}$ single crystals, with $0 \leq x<0.55$, obtained by using both identical and complementary experimental techniques. Although the proposed phase diagrams present discrepancies regarding the magnetic phase sequence and the ferroelectric properties for $0.15<x<0.25$, there is a good agreement concerning the phase sequence for $0.25<x<0.55$. 
Recently, a re-drawn $(x, \mathrm{~T})$ phase diagram of $\mathrm{Eu}_{1-x} \mathrm{Y}_{x} \mathrm{MnO}_{3}$, with $0 \leq x<0.55$, based on $\mathrm{X}$-ray diffraction, specific heat, dielectric constant and induced magnetization data, was published. ${ }^{31}$ Figure 7 shows the more recent proposed $(x, \mathrm{~T})$-phase diagram for this system. ${ }^{31}$

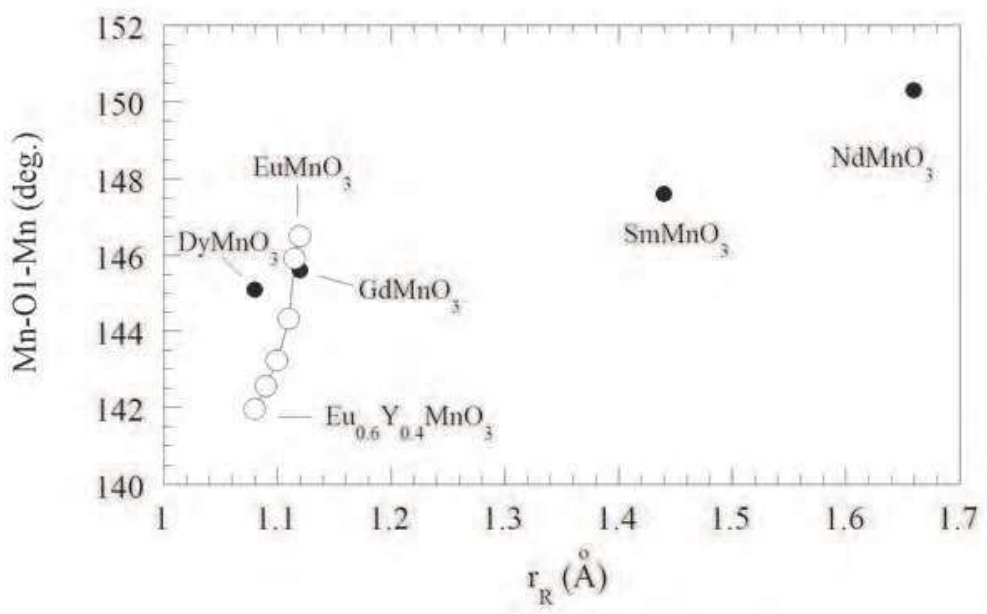

Fig. 6. Mn-O1-Mn bond angle as a function of the ionic radius of the A-site ion, for the $\mathrm{ReMnO}_{3}$, with $\mathrm{Re}=\mathrm{Nd}, \mathrm{Sm}, \mathrm{Eu}, \mathrm{Gd}$, Dy (closed circles), and for $\mathrm{Eu}_{1-x} \mathrm{Y}_{x} \mathrm{MnO}_{3}$ (open squares). Adapted figure from Ref. 31.

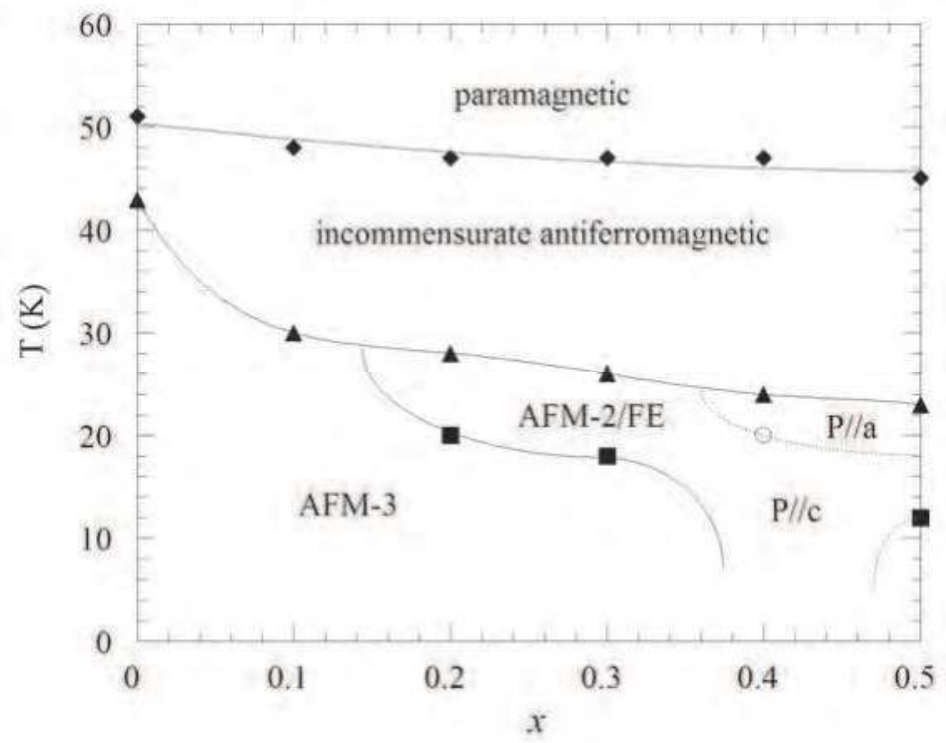

Fig. 7. $(x, \mathrm{~T})$ phase diagram of the $\mathrm{Eu}_{1-x} \mathrm{Y}_{x} \mathrm{MnO}_{3}$. The dashed lines stand for guessed boundaries. Adapted from Ref. 31. 
The phase boundaries were traced by considering the phase transition temperatures obtained from the set of data referred to above. Below the well-known sinusoidal incommensurate antiferromagnetic phase (hereafter designated by AFM-1), observed for all compounds, a re-entrant ferroelectric and antiferromagnetic phase (AFM-2) is stable for $x=$ $0.2,0.3$, and 0.5 . The ferroelectric character of this phase was established by $\mathrm{P}(\mathrm{E})$ data analysis. Conversely, the AFM-3 phase is non-polar. ${ }^{31}$ If the low temperature antiferromagnetic phase (AFM-3) were ferroelectric, as other authors reported previously, then, even though the coercive field had increased by decreasing temperatures, the remanent polarization would have increased accordingly. This is not confirmed by $\mathrm{P}(\mathrm{E})$ behaviour. In fact, the remanent polarization decreases to zero towards $\mathrm{T}_{\mathrm{AFM}-3}$, evidencing a low temperature non-polar phase. The decrease of the remanent polarization as the temperature decreases, observed for the compositions $x=0.2,0.3$, and 0.5 , can be associated with changes of both spin and lattice structures. As no ferroelectric behaviour was found for $0 \leq x<0.2$ down to $7 \mathrm{~K}$, and taking into account magnetization data, we have considered a unique weakly ferromagnetic phase (AFM-3). Our current data do not provide any other reasoning to further split this phase. The phase boundary between AFM-2 and AFM-3 phases for $0.3<x<0.5$ were not traced, since the experimental data do not indicate unambiguously whether a transition to a non ferroelectric phase occurs at temperatures below the lowest measured temperature. Moreover, it is not clear what are the phase boundaries associated with the polarization rotation from the $c$ to the $a$-axis, in the neighbouring of the composition $x=0.5$.

The aforementioned $(x, \mathrm{~T})$ phase diagram is significantly different from other earlier reported.24,26 Evidence for a unique non-ferroelectric low temperature AFM-3 phase is actually achieved. ${ }^{31}$

The origin of the ferroelectricity in these compounds is understood in the framework of the spin-driven ferroelectricity model.16 In these frustrated spin systems, the inverse Dzyaloshinsky-Morya interaction mechanism has been proposed. However, based on experimental results, the magnetic structure has been well established only for the compositions $x=0.4$ and 0.5 . For the other compositions, the magnetic structure is not yet determined. Moreover, the ferroelectric properties of the $\mathrm{Eu}_{1-x} \mathrm{Y}_{x} \mathrm{MnO}_{3}$ have been also studied by measurement of the electric current after cooling the sample under rather highapplied electric fields $(\mathrm{E}>1 \mathrm{kV} / \mathrm{cm})$. As it was shown in Refs. 29, 30 and 32, $\mathrm{Eu}_{1-x} \mathrm{Y}_{x} \mathrm{MnO}_{3}$ exhibits a rather high polarizability, which can prevent the observation of the spontaneous polarization.

\subsection{Experimental study and discussion}

In order to ascertain the correlation between crystal structure and spin arrangements referred to above different approaches can be realized. One way is to use high-resolution Xray diffraction using synchrotron radiation, to figure out the behaviour of structural parameters across the magnetic phase transitions. Very recently, a work on $\mathrm{Eu}_{1-x} \mathrm{Y}_{x} \mathrm{MnO}_{3}$ system was published showing the temperature behaviour of some structural parameters across the magnetic phase transitions. ${ }^{28}$ Anomalies observed in the lattice parameters and both octahedra bond angle and bond distances clear evidence spin rearrangements occurring at phase transition temperatures, which are in favour of a significant spin-lattice coupling in these materials. ${ }^{28}$ 
Another alternative way to figure out magnetic-induced ferroelectric ground states is to study the phonon behaviour across the magnetic phase transition through Raman spectroscopy. As the electric polarization arises from lattice distortions, the study of the spin-phonon coupling is particularly interesting in systems that present strong spin-lattice coupling, as it is the case of rare-earth magnetoelectric manganites. Consequently, from both fundamental point of view and technological applications, to comprehend spin-phonon coupling is a central research objective.

A variety of Raman scattering studies of orthorhombic rare-earth manganites, involving Pr, $\mathrm{Nd}$ and Sm, has evidenced a significant coupling between spins and phonons close and below the Néel temperature. ${ }^{34}$ We also note that the effect of the magnetic ordering is very weak in magnetoelectrics rare-earth manganites involving other rare-earth ions like $\mathrm{Gd}, \mathrm{Tb}$, Dy, Ho and Y. ${ }^{34}$ Since the change of the $\mathrm{GdFeO}_{3}$ distortion is accompanied with the change of the magnetic moment due to the rare-earth ions alteration, a comparative analysis of the coupling between spin degrees of freedom and phonons is a rather difficult task. This is not the case of the solid solution consisting of orthorhombic $\mathrm{Eu}_{1-x} \mathrm{Y}_{x} \mathrm{MnO}_{3}$ system $(0 \leq x \leq 0.5)$, since no magnetic contributions stem from europium and yttrium ions. In fact, the magnetic properties are entirely due to the manganese $3 d$ spins. In the absence of other effects, a direct relation of spin-phonon coupling with the $\mathrm{GdFeO}_{3}$ distortion can be achieved. We would like to emphasize that this distortion is a consequence of the Jahn-Teller cooperative effect and the tilting of the octahedra around the $a$-axis, which yield a lowering of the symmetry. As a consequence of this increasing lattice deformation, the orbital overlap becomes larger via the Mn-O1-Mn bond angle changing in turn the balance between ferromagnetic and antiferromagnetic exchange interaction. 50

The main goal is to understand how phonons relate with both lattice distortions and spin arrangements, and to determine their significance to stabilizing ferroelectric ground states.

In the following, we will present and discuss the main experimental results obtained in the aforementioned system, in the form of polycrystalline samples. Details of the sample processing and experimental method could be found in Refs. 69 and 38.

The Raman spectra were analyzed in the framework of the sum of independent damped harmonic oscillators, according to the general formula: ${ }^{70}$

$$
I(\omega, T)=(1+n(\omega, T)) \sum_{j=1}^{N} A_{o j} \frac{\omega \Omega_{\mathrm{oj}}^{2} \Gamma_{\mathrm{oj}}}{\left(\Omega_{\mathrm{oj}}^{2}-\omega^{2}\right)^{2}+\omega^{2} \Gamma_{\mathrm{oj}}^{2}},
$$

by fitting this equation to the experimental data. $n(\omega, T)$ stands for the Bose-Einstein factor, and $A_{o j}, \Omega_{o j}$ and $\Gamma_{o j}$ are the strength, wave number and damping coefficient of the $j$-th oscillator, respectively.

In the orthorhombic rare-earth manganites, the activation of the Raman modes is due to deviations from the ideal cubic perovskite structure. Factor group analysis of the $\mathrm{EuMnO}_{3}$ (with $\mathrm{Pbnm}$ orthorhombic structure) provides the following decomposition corresponding to the 60 normal vibrations at the $\Gamma$-point of the Brillouin zone:

$$
\begin{gathered}
\Gamma_{\text {acustic }}=B_{1 \mathrm{u}}+\mathrm{B}_{2 \mathrm{u}}+\mathrm{B}_{3 \mathrm{u}} \\
\Gamma_{\text {optical }}=\left(7 \mathrm{~A}_{\mathrm{g}}+7 \mathrm{~B}_{1 \mathrm{~g}}+5 \mathrm{~B}_{2 \mathrm{~g}}+5 \mathrm{~B}_{3 \mathrm{~g}}\right)_{\text {Raman-active }}+\left(8 \mathrm{~A}_{\mathrm{u}}+10 \mathrm{~B}_{1 \mathrm{u}}+8 \mathrm{~B}_{2 \mathrm{u}}+10 \mathrm{~B}_{3 \mathrm{u}}\right)_{\text {IR-active }}
\end{gathered}
$$

Since Raman-active modes should preserve the inversion centre of symmetry, the $\mathrm{Mn}^{3+}$ ions do not yield any contribution to the Raman spectra. From the polycrystallinity of the 
samples, the Raman spectra obtained involve all Raman-active modes. Earlier reports by Lavèrdiere $\mathrm{et} \mathrm{al}^{34}$ suggested that the more intense Raman bands are of $\mathrm{A}_{\mathrm{g}}$ and $\mathrm{B}_{2 \mathrm{~g}}$ symmetry. Therefore in our spectra, the $\mathrm{A}_{\mathrm{g}}$ and $\mathrm{B}_{2 \mathrm{~g}}$ modes are expected to be the more intense bands in $\mathrm{Eu}_{1-x} \mathrm{Y}_{x} \mathrm{MnO}_{3}$. As these modes are the most essential ones for our study, we are persuaded that by using ceramics instead of single crystals, no significant data are in fact lost in regard to the temperature dependence of the mode parameters.

Figure 8 shows the unpolarized Raman spectra of $\mathrm{Eu}_{1-x} \mathrm{Y}_{x} \mathrm{MnO}_{3}$, with $x=0,0.1,0.3,0.4$ and 0.5 , taken at room temperature.

The spectral signature of all $\mathrm{Eu}_{1-x} \mathrm{Y}_{x} \mathrm{MnO}_{3}$ (with $x \leq 0.5$ ) compounds is qualitatively similar in the $300-800 \mathrm{~cm}^{-1}$ frequency range, either in terms of frequency, linewidth or intensity. Their similarity suggests that they all crystallize into the same space group, and that the internal modes of the $\mathrm{MnO}_{6}$ octahedra are not very sensitive to Y-doping. This results is in excellent agreement with the quite similar structure, which is slightly dependent on Ycontent. 28,38 Nevertheless, a fine quantitative analysis of the spectra evidenced some subtle changes as $\mathrm{Y}$-concentration is altered. Some examples can be highlighted. The broad band emerging close to $520 \mathrm{~cm}^{-1}$ becomes more noticeable by increasing the yttrium concentration. The frequency of the band located near $364 \mathrm{~cm}^{-1}$ increases considerably with increasing $x$.

An earlier work by L. Martín-Carrón et al,45 regarding the frequency dependence of the Raman bands in some stoichiometric rare-earth manganites, has been used to assign the more intense Raman bands of each spectrum. The band at $613 \mathrm{~cm}^{-1}$ is associated with a JahnTeller symmetric stretching mode involving the $\mathrm{O} 2$ atoms (symmetry $\mathrm{B}_{2 \mathrm{~g}}$ ), 33,45,71,72 the band at $506 \mathrm{~cm}^{-1}$ to a bending mode (symmetry $\mathrm{B}_{2 \mathrm{~g}}$ ), the band at $484 \mathrm{~cm}^{-1}$ to a Jahn-Teller type asymmetric stretching mode involving also the $\mathrm{O} 2$ atoms (symmetry $\mathrm{A}_{\mathrm{g}}$ ), and the band at $364 \mathrm{~cm}^{-1}$ to a bending mode of the tilt of the $\mathrm{MnO}_{6}$ octahedra (symmetry $\mathrm{A}_{\mathrm{g}}$ ). ${ }^{45}$

From the mode assignment referred to above, it is now possible to correlate the $x$-dependence of the frequency of these Raman bands with the structural changes induced by the Y-doping. The more noticeable stretching modes in $\mathrm{ReMnO}_{3}$ are known to involve nearly pure Mn-O2 bond and they are found to be slightly dependent on the chemical pressure. In orthorhombic rare-earth manganites, the stretching modes change less than $5 \mathrm{~cm}^{-1}$, with the rare-earth ion substitution from La to Dy. 33 Figures 9 (a) and (c) show the frequency of the bands located close to $613 \mathrm{~cm}^{-1}$ and $484 \mathrm{~cm}^{-1}$, respectively, as a function of $x .^{38}$

The observed frequency changes of only $2 \mathrm{~cm}^{-1}$ when $x$ increases from 0 to 0.5 , correlates well with a weak dependence of the Mn-O2 bond lengths with $x .{ }^{38}$ The weak $x$-dependence of the frequency of these modes provides further evidence for a slight dependence of the $\mathrm{MnO}_{6}$ octahedron volume and $\mathrm{Mn}-\mathrm{O}$ bonds lengths on the Y-doping, in agreement with literature work on other rare-earth manganites.28,45 Contrarily, the modes $B$ and $T$ shown in Figures 9 (b) and (d) reveal a significant variation with $x, 10$ to $15 \mathrm{~cm}^{-1}$ when $x$ increases from 0 to 0.5 . This feature correlates well with the $x$-dependence of the tilt angle. ${ }^{38}$ The largest variations with $x$ is presented by the lower frequency $T$ mode, which is an external mode $\mathrm{A}_{\mathrm{g}}$ associated with the tilt mode of the $\mathrm{MnO}_{6}$ octahedra. A linear dependence of the frequency of $T$ mode in the tilt angle was in fact observed (see Fig. 7 of Ref 38). The slope found, $5 \mathrm{~cm}^{-1} / \mathrm{deg}$, is much less that the slope obtained for other orthorhombic manganites ( $23 \mathrm{~cm}^{-1} / \mathrm{deg}$ ). ${ }^{33}$ Mode $B$ is assigned to the bending mode $\mathrm{B}_{2 \mathrm{~g}}$ of the octahedra. ${ }^{33}$ The two broad shoulders observed at round $470 \mathrm{~cm}^{-1}$ and $520 \mathrm{~cm}^{-1}$ are likely the $\mathrm{B}_{2 \mathrm{~g}}$ in-phase $\mathrm{O} 2$ scissor-like and out-of-plane $\mathrm{MnO}_{6}$ bending modes. 


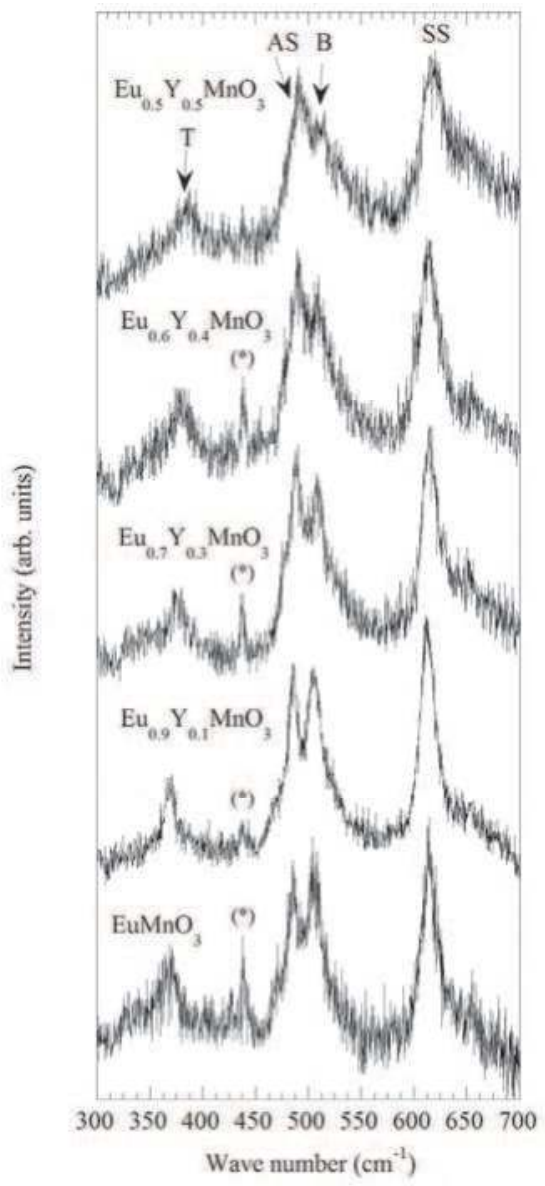

Fig. 8. Unpolarized Raman spectra of $\mathrm{Eu}_{1-x} \mathrm{Y}_{x} \mathrm{MnO}_{3}$, for $x=0,0.1,0.3,0.4$ and 0.5, recorded at room temperature. The laser plasma line is indicated by $\left(^{*}\right)$. Mode assignment: SS-symmetric stretching mode (symmetry $B_{2 g}$ ); AS - Jahn-Teller type asymmetric stretching mode (symmetry $\mathrm{A}_{\mathrm{g}}$ ); $\mathrm{B}$ - bending mode (symmetry $\mathrm{B}_{2 \mathrm{~g}}$ ); $\mathrm{T}$ - tilt mode of the $\mathrm{MnO}_{6}$ octahedra (symmetry $\mathrm{A}_{\mathrm{g}}$ ). Reprinted figure from Ref. 38. Copyright (2010) by the American Physical Society.

Let us address to the temperature dependence of the frequency of Raman active modes. Figure 10 shows the unpolarized Raman spectra of $\mathrm{EuMnO}_{3}$ and $\mathrm{Eu}_{0.5} \mathrm{Y}_{0.5} \mathrm{MnO}_{3}$, recorded at $200 \mathrm{~K}$ and $9 \mathrm{~K}$.

As it can be seen in Figure 10, the spectra at $200 \mathrm{~K}$ and $9 \mathrm{~K}$ show only very small changes in their profiles. Especially, no new bands were detected at low temperatures. The lack of emergence of infrared Raman-active bands, even for those compositions where the stabilization of a spontaneous ferroelectric order is expected, may have origin in two different mechanisms: either the inverse centre is conserved or the ferroelectric phases for $x$ $\geq 0.2$ are of an improper nature. 

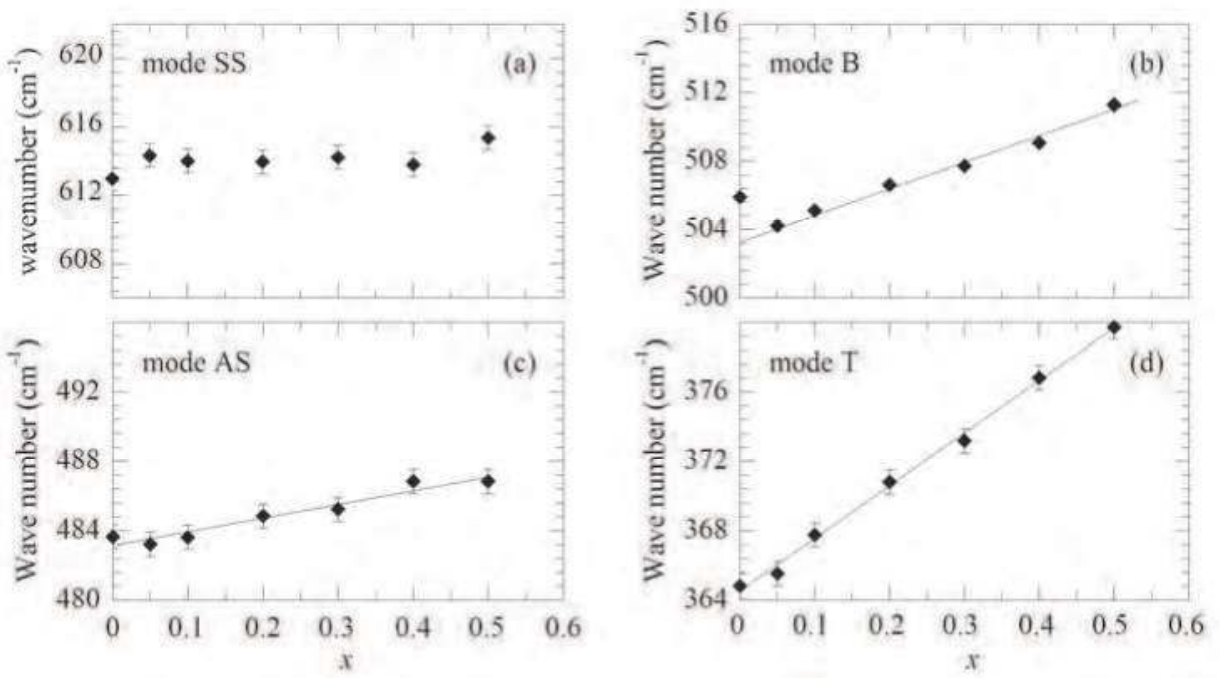

Fig. 9. Dependence of the frequency of the symmetric stretching mode (SS) (a), bending mode (B) (b), antisymmetric stretching mode (AS) (c) and tilt mode (T) (d), on the Y-content. The solid lines are guides for the eyes. Adapted from Ref. 38. Copyright (2010) by the American Physical Society.
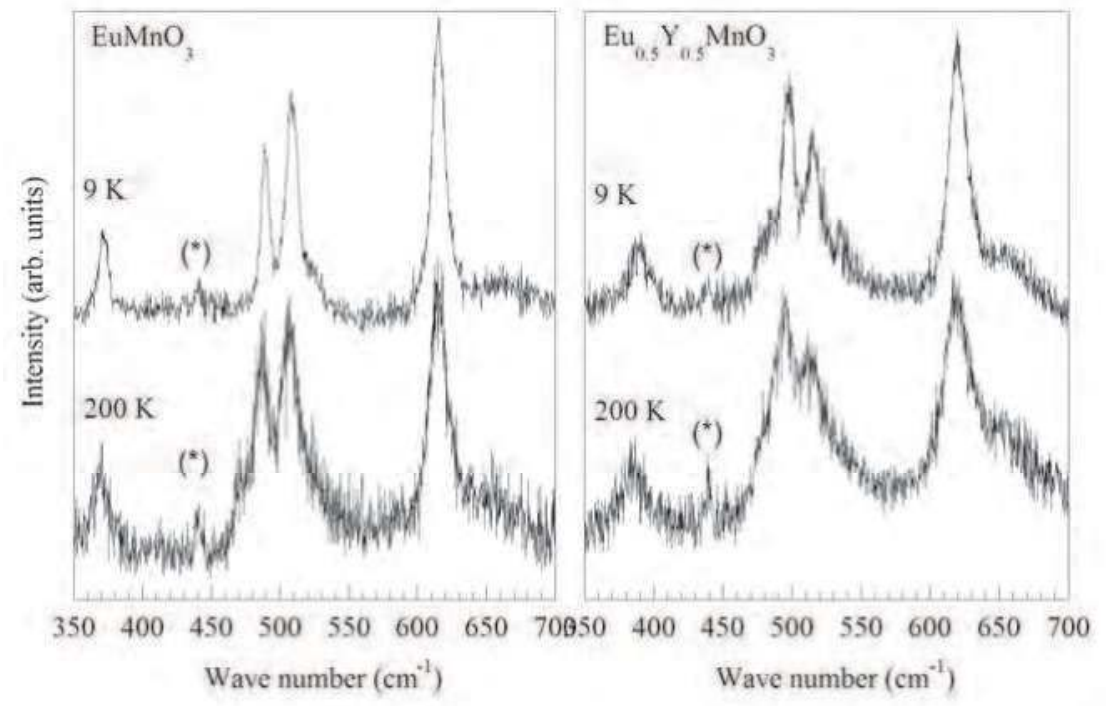

Fig. 10. The Raman spectra of $\mathrm{EuMnO}_{3}$ and $\mathrm{Eu}_{0.5} \mathrm{Y}_{0.5} \mathrm{MnO}_{3}$, recorded at 200 and at $9 \mathrm{~K}$. The laser plasma line is indicated by $\left(^{*}\right)$. Adapted from Ref. 38. 
Despite the absence of new Raman-active bands, an analysis of the spectra reveals striking anomalies in the temperature dependence of some phonon parameters throughout the magnetic phase transitions.

According to the spin-phonon coupling models, one should expect detectable changes in the phonon frequencies on entering the magnetic phases, reflecting the phonon renormalization, proportional to the spin-spin correlation function for the nearest $\mathrm{Mn}^{3+}$ spins. Aiming at searching for a spin-phonon coupling in $\mathrm{Eu}_{1-x} \mathrm{Y}_{x} \mathrm{MnO}_{3}$, we have followed the temperature dependence of the parameters characterizing the $\mathrm{MnO}_{6}$ Raman-active modes. We have found that the symmetric stretching mode (SS) close to $615 \mathrm{~cm}^{-1}$ is the most sensitive to the magnetic ordering. In fact, this mode senses any change in the geometrical parameters associated with the spontaneous orthorhombic strain $e$ and, so a strong coupling between the $\mathrm{B}_{2 \mathrm{~g}}$ symmetric stretching mode and the electronic degrees of freedom is ascertained. In other rare-earth manganites, like Ca-doped $\mathrm{PrMnO}_{3}$, this mode is so strongly coupled with the electronic system that it can be used to control a metal-insulator transition, by its coherent manipulation through selective mode excitation. ${ }^{73}$ The temperature dependence of the $B_{2 g}$ symmetric stretching mode frequency for different yttrium concentrations is shown in Figure 11, together with the insets, which reflect the temperature dependence of the corresponding linewidths.

In order to evaluate the effect of magnetic exchange interactions on the phonon behaviour, we start by defining the purely anharmonic temperature dependence of the frequency and of the linewidth of the different modes by the model: 74

$$
\omega(T)=\omega(0)+C\left(1-\frac{2}{e^{y}-1}\right)
$$

for the temperature dependence of the frequency of the transverse mode and: 74

$$
\Gamma(T)=\Gamma(0)\left(1-\frac{2}{e^{y}-1}\right)
$$

for the temperature dependence of its linewidth. In equations (8) and (9), $\omega(\mathrm{T})$ and $\omega(0)$ are the frequencies of the optical mode at the temperature $\mathrm{T}$ and $0 \mathrm{~K}$, respectively, $y=\frac{\hbar \omega_{o}}{2 k_{B} T}$ where $\omega_{\mathrm{o}}$ is the characteristic frequency of the mode, $\Gamma(\mathrm{T})$ and $\Gamma(0)$ are the linewidths of the mode at the temperature $\mathrm{T}$ and $0 \mathrm{~K}$, respectively. ${ }^{74}$ The solid lines in Figure 11 correspond to the best fit of these equations to the high-temperature range data ( $\mathrm{T}>100 \mathrm{~K})$, with the adjustable parameters $C, \omega(0), \Gamma(0)$ and $\omega_{0}$.

The results displayed in Figure 11 clearly show that for $\mathrm{Eu}_{1-x} \mathrm{Y}_{x} \mathrm{MnO}_{3}$, with $x=0,0.3$ and 0.4, only a faint frequency shift is observed at $T_{N}$ in the temperature dependence of the phonon frequency, from the normal anharmonic behaviour. Contrarily, a significant negative frequency shift is found for $x=0.2$, along with a negative and positive shifts observed for $x=0.1$ and $x=0.5$, respectively.

For all these compounds the shifts appear well above the onset of the magnetic order and consequently it is very unlikely that these effects are driven by any sort of long range spin ordering. It may be that the shifts emerge when the temperature allows for some kind of local spin ordering.

The frequency shift of a given phonon as a function of temperature, due to the spin-phonon coupling, is determined by the spin-spin correlation function: 68,75

$$
\omega=\omega_{o}+\gamma\left\langle\vec{S}_{i} \cdot \vec{S}_{j}\right\rangle
$$



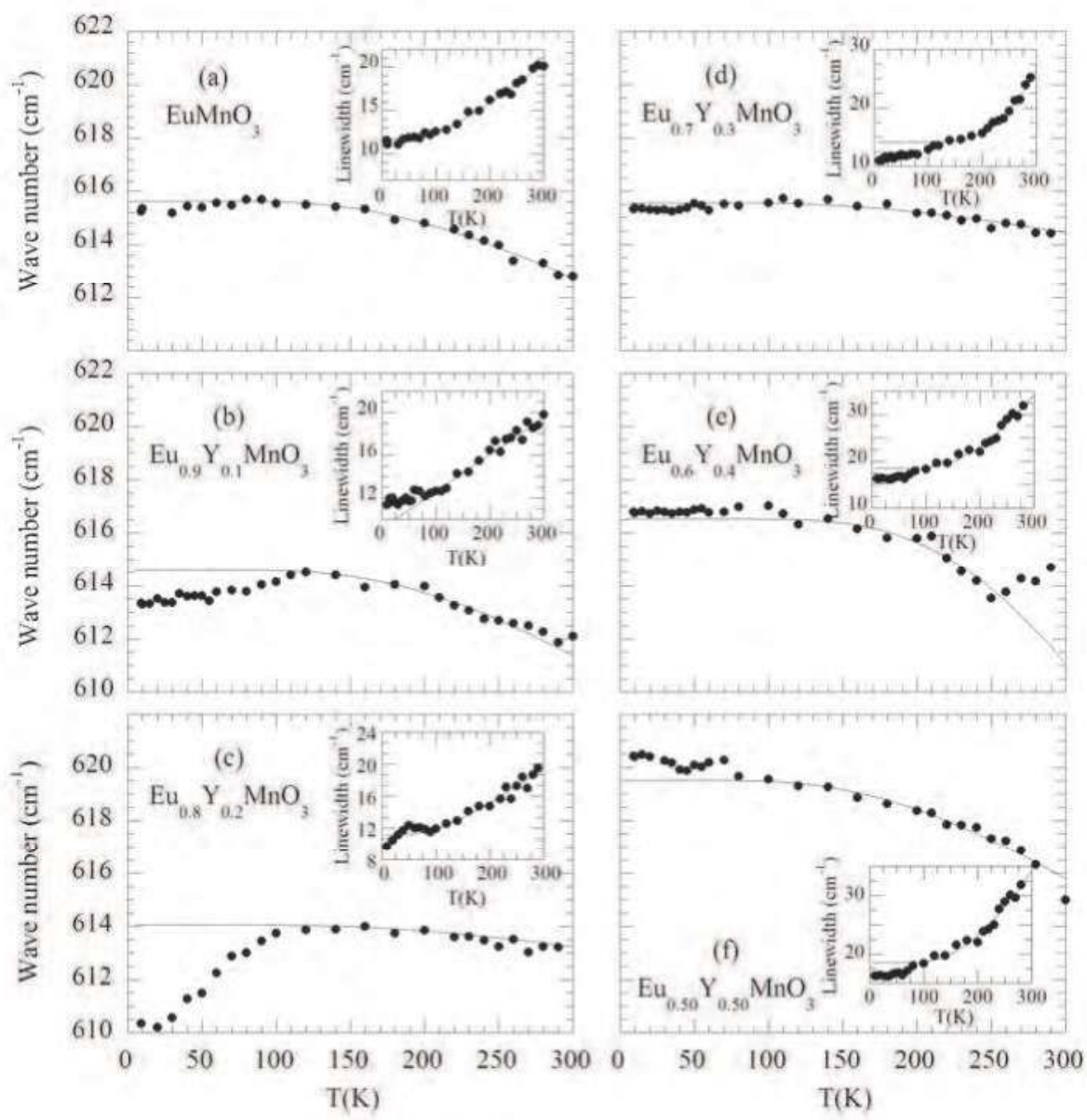

Fig. 11. Temperature dependence of the frequency of the $\mathrm{B}_{2 \mathrm{~g}}$ symmetric stretching mode (SS) for $\mathrm{Eu}_{1-x} \mathrm{Y}_{x} \mathrm{MnO}_{3}$, with $x=0,0.1,0.2,0.3,0.4$, and 0.5 . The insets show the temperature dependence of the damping coefficient of the same mode as a function of the temperature. The solid lines were obtained from the best fit of Eq. 8 and 9, respectively. Reprinted figure from Ref. 38. Copyright (2010) by the American Physical Society.

where $\omega$ is the renormalized phonon frequency at a fixed temperature, $\omega_{o}$ denotes the frequency in the absence of spin-phonon coupling, and $\gamma$ is the spin-phonon coupling constant.

When ferromagnetic and antiferromagnetic competitive interactions are present, it was proposed for the frequency shift: 75,76

$$
\omega-\omega_{o} \propto-R_{1}\left\langle\vec{S}_{i} \mid \vec{S}_{j}\right\rangle+R_{2}\left\langle\vec{S}_{i} \mid \vec{S}_{k}\right\rangle
$$

where $R_{1}$ and $R_{2}$ are spin dependent force constants of the lattice vibrations deduced as the squared derivatives of the exchange integrals with the respect to the phonon displacement. 
$R_{1}$ is associated with the ferromagnetic nearest neighbour and $R_{2}$ is associated with the antiferromagnetic next-nearest neighbour exchange.75 The magnetic properties are determined mainly by the exchange integrals, which depend on the number of ferromagnetic and antiferromagnetic interactions in the system. This model predicts negative or positive frequency shifts depending on the relative strength between the ferromagnetic and antiferromagnetic exchange interactions, associated with the normal mode being considered. On the grounds of the model presented to above, the Raman frequency shifts displayed in Figure 11 can be understood by assuming the coexistence of ferromagnetic and antiferromagnetic competitive exchange interactions, and spin-phonon coupling in $\mathrm{Eu}_{1-x} \mathrm{Y}_{x} \mathrm{MnO}_{3}$. As it has been assumed in current literature, we consider that the spin correlation functions of the nearest neighbours and the next-nearest neighbours have almost the same temperature dependence, ${ }^{37}$ and thus, we take the same correlation functions for $\left\langle\vec{S}_{i} \mid \vec{S}_{j}\right\rangle,\left\langle\vec{S}_{\mathrm{i}} \mid \vec{S}_{\mathrm{k}}\right\rangle$ and $\left\langle\vec{S}_{i} \mid \vec{S}_{k}\right\rangle$. Moreover, as we are dealing with the same eigenmode, we also assume constant values for $R_{1}$ and $R_{2}$. So, Equation (11) can be written as follows:

$$
\omega-\omega_{o} \propto\left(R_{2}-R_{1}\right)\left\langle\vec{S}_{i} \mid \vec{S}_{j}\right\rangle .
$$

For $x=0,0.1$ and 0.2 , the Raman shift is negative, increasing as the $x$ value increases up to 0.2 , where it takes its maximum value. This means that the difference $R_{2}-R_{1}$ becomes more negative with $x$ up to 0.2 . For $x=0.3$, the Raman frequency shift is also negative, but with a small value. On the other hand, for $x=0.4$ and 0.5 a clear positive Raman shift is observed. It is worthwhile to stress that the weak ferromagnetic character of the compounds with $x$ between 0 and 0.2 cannot be explain by this model, as it is associated with the number of the exchange integrals and not with the second derivatives to the phonon displacements, represented by the $R_{1}$ and $R_{2}$ coefficients. This in good agreement with fact that the ferromagnetic features in these compounds arise below $45 \mathrm{~K}-28 \mathrm{~K}$, depending on $x$ (see phase diagram in Figure 5 of Ref. 50), which is far below $100 \mathrm{~K}$ where the spin-phonon coupling mechanism emerges.

Except for $\mathrm{EuMnO}_{3}$, the linewidth deviates, around $100 \mathrm{~K}$, from the purely anharmonic temperature dependence behaviour. For $x=0.20$, the linewidth presents a further anomaly at $\mathrm{T}_{\mathrm{N}} \approx 50 \mathrm{~K}$ where the temperature derivative of the wave number is maximum.

In order to search for further corroboration of the spin-phonon coupling, we have also studied the temperature behaviour of the lattice mode associated with the rotational $\mathrm{A}_{\mathrm{g}}$ mode of the $\mathrm{MnO}_{6}$ octahedra, which scales directly with the Mn-O1-Mn bond angle. Figure 12 shows the results obtained for $x=0.3$ and 0.4 . Both anomalies at $\mathrm{T}_{\mathrm{N}}$, for $x=0.3$ and 0.4 , and at $\mathrm{T}_{\mathrm{AFM}-2}$ (only for $x=0.4$ ) and deviations from the normal anharmonic behaviour, for both $x=0.3$ and 0.4 , entirely corroborates the role of spin-phonon coupling mechanism in $\mathrm{Eu}_{1-x} \mathrm{Y}_{x} \mathrm{MnO}$. It is still worth noting, that the sign of the shifts is fully consistent with the magnetic character of both compositions. The negative (positive) shift obtained for $x=0.3$ $(x=0.4)$ confirms the relative predominance of the ferromagnetic (antiferromagnetic) exchanges against the antiferromagnetic (ferromagnetic) ones. This feature is in favour of an increasing range of stability of the ferroelectric ground state as the yttrium concentration increases. The different configuration of the low temperature part of the $\mathrm{Eu}_{1-x} \mathrm{Y}_{x} \mathrm{MnO}_{3}$ phase diagram is in good agreement with the very distinct behaviour of the Mn-O-Mn bond angle versus the average A-site size, when compared with the one of other magnetoelectric rareearth manganites (see Fig. 6). 

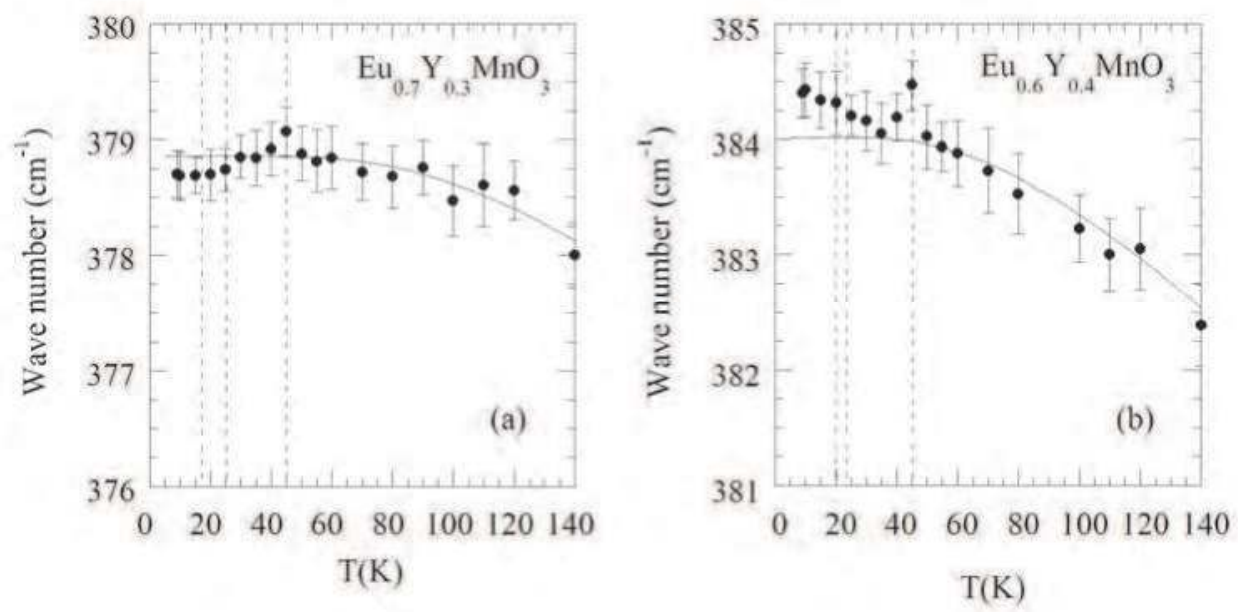

Fig. 12. Temperature dependence of the frequency of the $\mathrm{A}_{g}$ external mode $(\mathrm{T})$ for $\mathrm{Eu}_{1-}$ ${ }_{x} \mathrm{Y}_{x} \mathrm{MnO}_{3}$, with $x=0.3$ and 0.4 . The solid lines were obtained from the best fit of Eq. 8 and 9, respectively. Reprinted figure from Ref. 38. Copyright (2010) by the American Physical Society.

\section{Conclusions}

The experimental results obtained in $\mathrm{Eu}_{1-x} \mathrm{Y}_{x} \mathrm{MnO}_{3}$, with $0 \leq x \leq 0.5$, using Raman spectroscopy noticeably reveal the existence of spin-phonon coupling in this system: shifts of the frequency of the normal modes relative to anharmonic temperature dependence, energy transfer mechanisms between modes, anomalies in the mode parameters both above and below to $\mathrm{T}_{\mathrm{N}}$. This is most evidenced by the symmetric stretching and tilt modes associated with $\mathrm{MnO}_{6}$ octahedra.

Despite the existence of the spin-phonon coupling for all $x$ values studied, spin-phonon coupling is not a sufficient condition to the emergence of ferroelectricity. As it can be observed in $(\mathrm{x}, \mathrm{T})$ phase diagram, ferroelectricity occurs only above $x=0.15$, which means that other mechanisms are required. These mechanisms were theoretically established on the grounds of the model presented to above. ${ }^{50}$ The relative magnitude of the Hamiltonian terms shape the $(x, T)$ phase diagram, as $J_{2}$ increases. In fact, the emergence of the re-entrant ferroelectric phase stems from the increasing magnitude of the AFM against the FM interactions and of DM interaction. $30,38,50$

An alternative way towards ferroelectricity is to apply an external magnetic field. ${ }^{77}$ Experiments carried out in $\mathrm{Eu}_{1-x} \mathrm{Y}_{x} \mathrm{MnO}_{3}$ under magnetic field up to $55 \mathrm{~T}$, can induce magnetic transitions to a multiferroic phase. ${ }^{77}$ This same effect was also observed in $\mathrm{GdMnO}_{3}$, but for much lower values of the external magnetic field. ${ }^{17}$ Figure 13 shows the frequency of the $\mathrm{A}_{g}$ external mode as a function of the magnetic field, recorded at $10 \mathrm{~K}$. 
The anomalies mark the emergence of ferroelectriciy for a magnetic field of $3 \mathrm{~T}$, in good agreement with earlier published results. ${ }^{17}$ This in favour for the presence of a significant spin-phonon coupling in $\mathrm{GdMnO}_{3}$.

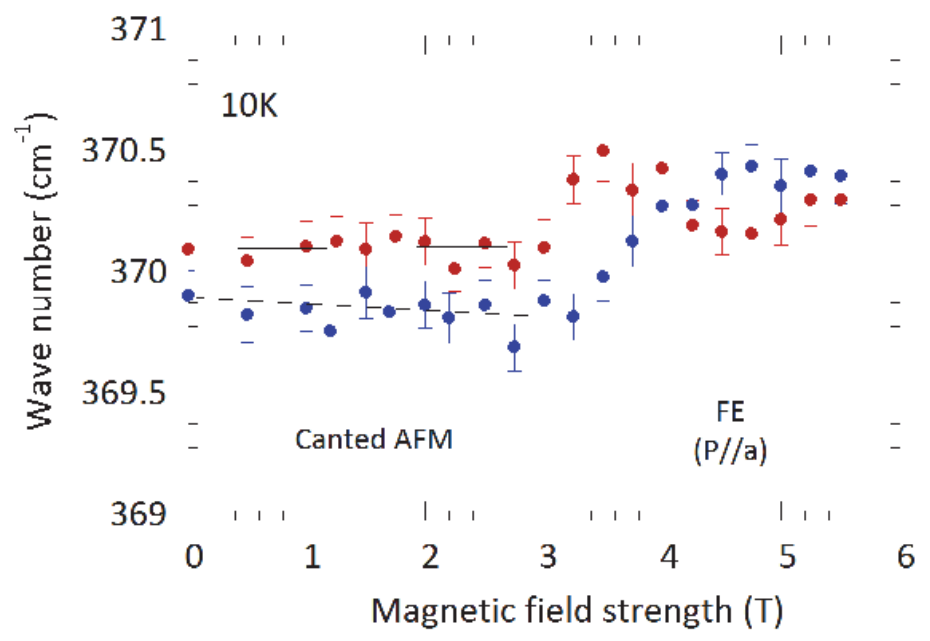

Fig. 13. Magnetic field dependence of the $\mathrm{A}_{\mathrm{g}}$ external mode frequency of $\mathrm{GdMnO}_{3}$, at $10 \mathrm{~K}$. Blue solid circles: increasing magnetic field strength; red solid circles: decreasing magnetic field strength. The vertical dashed lines mark the phase boundaries between the canted antiferromagnetic and the ferroelectric (P//a) phases, according Ref. 17.

Our results also yield the existence of spin-phonon coupling even above $\mathrm{T}_{\mathrm{N}}$, which in favour of a coupling between phonons and magnetic ordering in the paramagnetic phase. In fact, our experimental results correlate with terahertz transmittance data in $\mathrm{Eu}_{1-}$ ${ }_{\mathrm{x}} \mathrm{Y}_{\mathrm{x}} \mathrm{MnO}_{3} .{ }^{27,36}$ They yield the existence of electromagnons for $x=0.20,0.30$ and 0.50 , yielding information on the static dielectric constant below $\mathrm{T}_{1}$, and evidencing a broad background absorption observed up to $\mathrm{T}_{\mathrm{N}}+50 \mathrm{~K} .27,36$ We have also observed a frequency deviation from purely anharmonic behaviour, well above $T_{N}$, associated with spinphonon coupling. Laverdière et al ${ }^{34}$ have observed a softening for $\mathrm{A}_{\mathrm{g}}$ and $\mathrm{B}_{2 \mathrm{~g}}$ stretching modes in, e.g. $\mathrm{NdMnO}_{3}$ and $\mathrm{DyMnO}_{3}$, starting well above $\mathrm{T}_{\mathrm{N}}$, which they relate to a small $\mathrm{Mn}-\mathrm{O}$ expansion. Since manganites have peculiar local electronic and magnetic structures, it is also reasonable to associate this behaviour with local magnetic fluctuations, like in rare-earth nickelates.78-80 Aguilar et al27 have associated the existence of the terahertz background absorption in the paramagnetic phase with phonon-electromagnon coupling. We have reconsidered the correlation between the total spectral weight below $140 \mathrm{~cm}^{-1}$, depicted in Fig 2 of Ref. 27, and the frequency shift calculated from our results for $\mathrm{x}=$ 0.20. The results are shown in Figure 14.

This result evidences a mechanism that involves spin-spin interactions, since the shift of the measured frequency (proportional to $\vec{S}_{i} \cdot \vec{S}_{j}$ ) for $x=0.2$ scales with the spectral weight displayed in Figure 2 of the Ref. 27, in the temperature range $50 \mathrm{~K}-100 \mathrm{~K}$. The spectral 
weight, calculated from the sample with $x=0.25$, is reported to be proportional to the phonon-electromagnon coupling in the paramagnetic phase, and it is also proportional to $\vec{S}_{i} \cdot \vec{S}_{j}$.

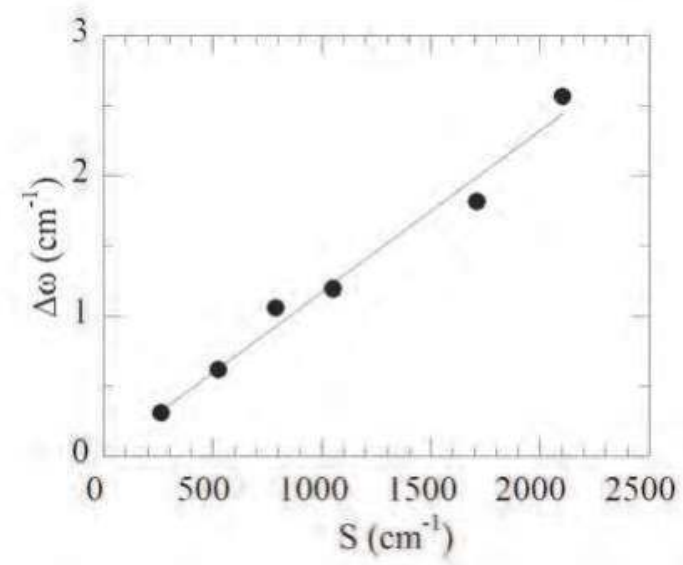

Fig. 14. Frequency deviation from the extrapolated temperature behaviour for $\mathrm{T}>100 \mathrm{~K}$, of the symmetric stretching mode of $\mathrm{Eu}_{0.8} \mathrm{Y}_{0.2} \mathrm{MnO}_{3}$, versus the spectral weight calculated from the terahertz data, below $140 \mathrm{~cm}^{-1}$, for the sample $\mathrm{Eu}_{0.75} \mathrm{Y}_{0.25} \mathrm{MnO}_{3}$ (Ref. 27). The straight line is a guide for the eyes. Copyright (2010) by the American Physical Society.

\section{Acknowledgements}

We would like to deeply thank Prof. Maria Renata Chaves and Dr. Jens Kreisel for very helpful discussions and distinct contributions. We thank Welberth Santos Ferreira for his technical assistance. We thank Fundação para a Ciência e Tecnologia through the Project No. PTDC/CTM/67575/2006.

\section{References}

[1] For a review on this topic see the special issues: The European Physical Journal B 71 (2009); J.Phys.: Condens. Matter 20 (2008), and references wherein presented.

[2] W. Eerenstein, N. D. Mathur, J. F. Scott, Nature 442, 759 (2006)

[3] M. Bibes and A. Barthelemy, IEEE Trans. Electron. Devices 54, p1003 (2007).

[4] B. B. van Aken, T. T. Palstra, M. Filipetti, and N. A. Spaldin, Nat. Mater. 3, 164 (2004).

[5] H. Béa, M. Bibes, and S. Cherifi, Appl. Phys. Lett. 89, 242114 (2006).

[6] R. Ramesh and N. A. spaldin, Nat. Mater. 6, 21 (2007).

[7] P. Rovillain, R. de Sousa, Y. Gallais, A. Sacuto, M. A. Méasson, D. Colson, A. Forget, M. Bibes, A. Barthélémy, M. Cazayous, Nat. Mater. 9, 975 (2010).

[8] Marin Alexe, Dietrich Hesse, Nat. Communications 2, 256 (2011). 
[9] Q. He, Y. -H. Chu, J. T. Heron, S. Y. Yang, W. I. Liang, C.Y. Kuo, H. J. Lin, P. Yu, C. W. Liang, R. J. Zeches, W. C. Kuo, J. Y. Juang, C. T. Chen, E. Arenholz, A. Scholl, and R. Ramesh, Nat. Communications 2, 225 (2011).

[10] N. Choudhury, L. Walizer, S. Lisenkov, L. Bellaiche, Nature 470, 513-517 (2011).

[11] S. M. Wu, Shane A. Cybart, P. Yu, M. D. Rossell, J. X. Zhang, R. Ramesh, R. C. Dynes, Nat. Mater. 9, 756 (2010).

[12] N. A. Hill, J. Phys. Chem. B 104, 6694 (2000).

[13] T. Kimura, S. Ishihara, H. Shintani, T. Arima, K. T. Takahashi, K. Ishizaka, and Y. Tokura, Phys. Rev. B 68, 060403 (2003).

[14] H. Katsura, N. Nagaosa, and A. V. Balatsky, Phys. Rev. Lett. 95, 057205 (2005).

[15] M. Mostovoy, Phys. Rev. Lett. 96, 067601 (2006).

[16] A. Sergienko and E. Dagotto, Phys. Rev. B 73, 094434 (2006).

[17] T. Goto, T. Kimura, G. Lawes, A. P. Ramirez, and Y. Tokura, Phys. Rev. Lett. 92, 257201 (2004).

[18] T. Kimura, G. Lawes, T. Goto, Y. Tokura, and A. P. Ramirez, Phys. Rev. B 71, 224425 (2005).

[19] T. Kimura, T. Goto, H. Shintani, K. Ishizaka, T. Arima, and Y. Tokura, Nature 426, 55 (2033).

[20] O. Prokhnenko, R. Feyerherm, M. Mostovoy, N. Aliouane, E. Dudzik, A. U. B. Wolter, A. Maljuk, and D. N. Argyriou, Phys. Rev. Lett. 99, 177206 (2007).

[21] T. Arima, T. Goto, Y. Yamasaki, S. Miyasaka, K. Ishii, M. Tsubota, T. Inami, Y. Murakami, and Y. Tokura, Pjys. Rev. B 72, 100102 (2005).

[22] K. Noda, S. Nakamura, J. Nagayama, and H. Kuwahara, J. Appl. Phys 97, 10C103 (2005).

[23] J. Baier, D. Meier, K. Berggold, J. Hemberger, A. Balbashov, J. A. Mydosh, and T. Lorenz, Pjys. Rev. B 73, 100402 (2006).

[24] J. Hemberger, F. Schrettle, A. Pimenov, P. Lunkenheimer, V. Yu. Ivanov, A. A. Mukhin, A. M. Balbashov, and A. Loidl, Phys. Rev. B 75, 035118 (2007).

[25] Y. Takahashi, Y. Yamasaki, N. Kida, Y. Kaneko, T. Arima, R. Shimano, and Y. Tokura, Phys. Rev. B 79, 214431 (2009).

[26] Y. Yamasaki, S. Miyasaka, T. Goto, H. Sagayama, T. Arima, and Y. Tokura, Phys. Rev. B 76, 184418 (2007).

[27] R. Valdés Aguilar, A. B. Sushkov, C. L. Zhang, Y. J. Choi, S.-W. Cheong, and H. D. Drew. Phys. Rev. B 76, 060404 (2007).

[28] J. Agostinho Moreira, A. Almeida, W. S. Ferreira, J. P. Araújo, A. M. Pereira, M. R. Chaves, M. M. R. Costa, V. A. Khomchenko, J. Kreisel, D. Chernyshov, S. M. F. Vilela, and P. B. Tavares, Phys. Rev. B 82, 094418 (2010).

[29] J. Agostinho Moreira, A. Almeida, W. S. Ferreira, M. R. Chaves, B. Kundys, R. Ranjith, W. Prellier, S. F. M. Vilela, and P. B. Tavares, J. Appl. Phys. 107, 024108 (2010).

[30] J. Agostinho Moreira, A. Almeida, W. S. Ferreira, M. R. Chaves, J. P. Araújo, A. M. Pereira, S. F. M. Vilela, and P. B. Tavares, J. Phys.: Conden. Matter 22, 125901 (2010). 
[31] J. Agostinho Moreira, A. Almeida, W. S. Ferreira, M. R. Chaves, J. B. Oliveira, J. M. M. da Silva, M. A. Sá, S. F. M. Vilela, and P. B. Tavares, Solid. State Commun. 151, 368 (2011).

[32] J. Agostinho Moreira, A. Almeida, W. S. Ferreira, M. R. Chaves, B. Kundys, R. Ranjith, W. Prellier, S. F. M. Vilela, and P. B. Tavares, J. Phys.: Condens. Matter 21, 446002 (2009).

[33] M. N. Iliev, M. V. Abrashev, J. Laverdière, S. Jandl, M. M. Gospodinov, Y.-Q. Wang, and Y.-Y. Sun, Phys. Rev. B 73, 064302 (2006).

[34] J. Laverdière, S. Jandl, A. A. Mukhin, V. Yu. Ivanov, V. G. Ivanov, and M. N. Iliev, Phys. Rev. B 73, 214301 (2006).

[35] S. Issing, A. Pimenov, V. Yu. Ivanov, A. A. Mukhin, and J. Geurts, Phys. Rev. B 81, 024304 (2010).

[36] Pimenov, A. Loidl, A. A. Mukhin, V. D. Travkin, V. Yu. Ivanov, and A. M. Balbashov, Phys. Rev. B 77, 014438 (2008).

[37] W. S. Ferreira, J. Agostinho Moreira, A. Almeida, M. R. Chaves, J. P. Araújo, J. B. Oliveira, J. M. Machado Da Silva, M. A. Sá, T. M. Mendonça, P. Simeão Carvalho, J. Kreisel, J. L. Ribeiro, L. G. Vieira, P. B. Tavares, and S. Mendonça, Phys. Rev. B 79, 054303 (2009).

[38] J. Agostinho Moreira, A. Almeida, W. S. Ferreira, J. P. Araújo, A. M. Pereira, M. R. Chaves, J. Kreisel, S. M. F. Vilela, and P. B. Tavares, Phys. Rev. B 81, 054447 (2010).

[39] S. Kamba, D. Nuzhnyy, M. Savinov, J. Sebek, J. Petzelt, J.Prokleska, R. Haumont, J. Kreisel, Phys. Rev. B 75, 024403 (2007).

[40] R. Haumont, J. Kreisel, P. Bouvier, F. Hippert, Phys. Rev. B 73, 132101 (2006).

[41] P. Hermet, M. Goffinet, J. Kreisel, P. Ghosez, Phys. Rev. B 75, 220102 (2007).

[42] K. H. J. Buschow and F. R. de Boer, Physics of Magnetism and Magnetic Materials. Kluber Academic Press. (2003).

[43] G. F. Dionne. Magnetic Oxides. Springer. (2009).

[44] M. V. Abrashev, J. Bäckström, L. Börjesson, M. Pissas, N. Kolev, and M. N. Iliev, Phys. Rev. B 64, 144429 (2001).

[45] L. Martín-Carrón, A. de Andrés, M. J. Martínez-Lope, M. T. Casais, and J. A. Alonso, Phys. Ver. B 66, 174303 (2002).

[46] L. D. Landau and E. Lifshitz, Electrodynamics of Continuous Media. ButterworthHeinemann. Oxford (2002).

[47] Dzyaloshinskii, J. Phys. Chem. Solids 4, 241 (1958).

[48] T. Moriya, Phys. Rev. 120, 91 (1960).

[49] H. Katsura, N. Nagaosa, and A. V. Balatsky, Phys. Rev. Lett. 95, 057205 (2005).

[50] M. Mochizuki and N. Furukawa, Phys. Rev. B 80, 134416 (2009).

[51] Y. Yamasaki, H. Sagayama, T. Goto, M. Matsuura, K. Hirota, T. Arima, and Y. Tokura, Phys. Rev. Lett. 98, 147204 (2007).

[52] H. J. Xiang, Su-Huai Wei, M.-H. Whangbo, and Juarez L. F. da Silva, Phys. Rev. Lett. 101, 037209 (2008). 
[53] M. Kenzelmann, A. B. Harris, S. Jonas, C. Broholm, J. Schefer, S. B. Kim, C. L. Zhang, S.-W. Cheong, O. P. Vajk, and J. W. Lynn, Phys. Rev. Lett. 95, 087206 (2005).

[54] R. Feyerherm, E. Dudzik, N. Aliouane, and D. N. Argyriou, Phys. Rev. B 73, 180401 (2006).

[55] T. Goto, Y. Yamasaki, H. Watanabe, T. Kimura, and Y. Tokura, Phys. Rev. B 72, 220403 (2005).

[56] Y. Yamasaki, H. Sagayama, N. Abe, T. Arima, K. Sasai, M. Matsuura, K. Hirota, D. Okuyama, Y. Noda, and Y. Tokura, Phys. Rev. Lett. 101, 097204 (2008).

[57] V. Yu. Ivanov, A. A. Mukin, V. D. Travkin, A. S. Prokhorov, A. M. Kadomtsev, Yu. F. Popov, G. P. Vorobev, K. I. Kamilov, and A. M. Balbashov, J. Magn. Magn. Mater. 300, e130 (2006).

[58] V. Yu. Ivanov, A. A. Mukin, V. D. Travkin, A. S. Prokhorov, Yu. F. Popov, A. M. Kadomtsev, G. P. Vorob'ev, K. I. Kamilov, and A. M. Balbashov, Phys. Status Solidi B 243, 107 (2006).

[59] Yoshimori, J. Phys. Soc. Jpn. 14, 807 (1959).

[60] T. A. Kaplan, Phys. Rev. 116, 888 (1959).

[61] J. Villain, J. Phys. Chem. Solids 11, 303 (1959).

[62] S. Dong, R. Yu, S. Yunoki, J.-M. Liu, and E. Dagotto, Phys. Rev. B 78, 155121 (2008).

[63] T. Moriya, Phys. Rev. Lett. 4, 228 (1960).

[64] V. Skumryev, F. Ott, J. M. D. Coey, A. Anane, J. -P. Renard, L. Pinsard_Gaudart, and A. Revcolevschi, Euro. Phys. J. B 11, 40 (1999).

[65] J. Hemberger, T. Rudolf, H.-A. Krug von Nidda, F. Mayr, A. Pimenov, V. Tsurkan, and A. Loidl, Phys. Rev. Lett. 97, 087204 (2006).

[66] Jiasi Xu, Jung H. Park, and Hyun M. Jang, Phys. Rev. B 75, 012409 (2007).

[67] E. Granado, A. Garca, J. A. Sanjurjo, C. Rettori, I. Torriani, F. Prado, R. D. Sanchez, A. Caneiro, and S. B. Oseroff, Phys. Rev. B 60, 11879 (1999).

[68] D. J. Lockood, and M. G. Cottam, J. Appl. Phys. 64, 5876 (1988).

[69] J. Agostinho Moreira, A. Almeida, W. S. Ferreira, M. R. Chaves, J. B. Oliveira, J. M. M. da Silva, M. A. Sá, S. F. M. Vilela, and P. B. Tavares, J. Electro. Ceram. 25, 203 (2010).

[70] W. Hayes and R. Loudon. Scattering of light by crystals. John Wiley and Sons (1978).

[71] M. N. Iliev, M. V. Abrashev, H. G. Lee, V. N. Popov, Y. Y. Sun, C. Thomsen, R. L. Meng, and C. W. Chu, Phys. Rev. B 57, 2872(1998).

[72] V. A. Amelitchev, B. Güttler, O. Yu. Gorbenko, A. R. Kaul, A. A. Bosak, and A. Yu. Ganin, Phys. Rev. B 63, 104430 (2001).

[73] M. Rini, R. Tobey, N. Dean, J. Itatani, Y. Tomioka, Y. Tokura, R. W. Schoenlein, and A. Cavalleri, Nature 449, 72 (2007).

[74] M. Balkanski, R. F. Wallis, and E. Haro, Phys. Rev. B 28, 1928 (1983).

[75] W. Baltensperger and J. S. Helman, Helv. Phys. Acta 41, 668 (1968).

[76] K. Wakamura and T. Arai, J. Appl. Phys. 63, 5824 (1988).

[77] M. Tokunaga, Y. Yamasaki, Y. Onose, M. Mochizuki, N. Furukawa, and Y. Tokura, Phys. Rev. Lett. 103, 187202 (2009).

[78] J. M. Wesselinowa, and A. T. Apostolov, J. Phys.: Condens. Matter 8, 473 (1996). 
[79] C. Girardot, J. Kreisel, S. Pignard, N. Caillault, and F. Weiss, Phys. Rev. B 78, 104101 (2008).

[80] G. Catalan, Phase Transitions 81, 729 (2008). 


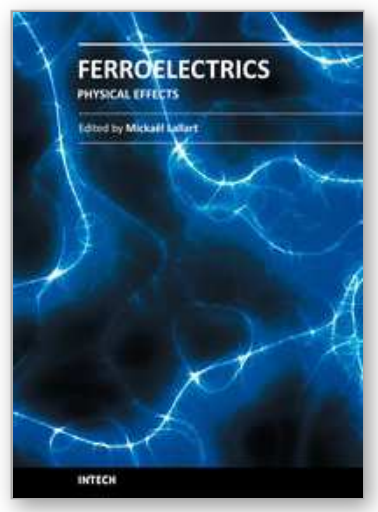

\author{
Ferroelectrics - Physical Effects \\ Edited by Dr. MickaÃ «l Lallart
}

ISBN 978-953-307-453-5

Hard cover, 654 pages

Publisher InTech

Published online 23, August, 2011

Published in print edition August, 2011

Ferroelectric materials have been and still are widely used in many applications, that have moved from sonar towards breakthrough technologies such as memories or optical devices. This book is a part of a four volume collection (covering material aspects, physical effects, characterization and modeling, and applications) and focuses on the underlying mechanisms of ferroelectric materials, including general ferroelectric effect, piezoelectricity, optical properties, and multiferroic and magnetoelectric devices. The aim of this book is to provide an up-to-date review of recent scientific findings and recent advances in the field of ferroelectric systems, allowing a deep understanding of the physical aspect of ferroelectricity.

\title{
How to reference
}

In order to correctly reference this scholarly work, feel free to copy and paste the following:

J. Agostinho Moreira and A. Almeida (2011). Coupling Between Spins and Phonons Towards Ferroelectricity in Magnetoelectric Systems, Ferroelectrics - Physical Effects, Dr. MickaÃ«l Lallart (Ed.), ISBN: 978-953-307-4535, InTech, Available from: http://www.intechopen.com/books/ferroelectrics-physical-effects/coupling-betweenspins-and-phonons-towards-ferroelectricity-in-magnetoelectric-systems

\section{INTECH}

open science | open minds

\section{InTech Europe}

University Campus STeP Ri

Slavka Krautzeka 83/A

51000 Rijeka, Croatia

Phone: +385 (51) 770447

Fax: +385 (51) 686166

www.intechopen.com

\section{InTech China}

Unit 405, Office Block, Hotel Equatorial Shanghai

No.65, Yan An Road (West), Shanghai, 200040, China 中国上海市延安西路65号上海国际贵都大饭店办公楼 405 单元

Phone: +86-21-62489820

Fax: +86-21-62489821 
(C) 2011 The Author(s). Licensee IntechOpen. This chapter is distributed under the terms of the Creative Commons Attribution-NonCommercialShareAlike-3.0 License, which permits use, distribution and reproduction for non-commercial purposes, provided the original is properly cited and derivative works building on this content are distributed under the same license. 